\title{
From outburst to quiescence: the decay of the transient AXP XTE J1810-197
}

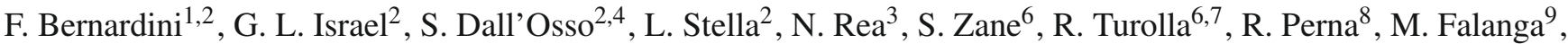 \\ S. Campana ${ }^{5}$, D. Götz ${ }^{9}$, S. Mereghetti ${ }^{10}$, and A. Tiengo ${ }^{10}$ \\ 1 Università degli Studi di Roma "Tor Vergata" via Orazio Raimondo 18, 00173 Roma, Italy \\ 2 INAF - Osservatorio Astronomico di Roma, via Frascati 33, 00040 Monteporzio Catone (Roma), Italy \\ e-mail: bernardini@oa-roma.inaf.it \\ 3 University of Amsterdam, Astronomical Institute "Anton Pannekoek", Kruislaan 403, 1098 SJ, Amsterdam, The Netherlands \\ 4 Dipartmento di Fisica "Enrico Fermi”, Universitá di Pisa, Largo B. Pontecorvo 3, 56127 Pisa, Italy \\ 5 INAF - Osservatorio Astronomico di Brera, via Bianchi 46, 23807 Merate (Lc), Italy \\ 6 Mullard Space Science Laboratory University College of London Holmbury St Mary, Dorking, Surrey, RH5 6NT, UK \\ 7 Department of Physics, University of Padova, via Marzolo 8, 35131 Padova, Italy \\ 8 JILA, Univ. of Colorado, Boulder, CO 80309-0440, USA \\ 9 CEA Saclay, DSM/DAPNIA/Service d'Astrophysique, 91191 Gif-sur-Yvette, France \\ ${ }^{10}$ INAF - Istituto di Astrofisica Spaziale e Fisica Cosmica Milano, Via Edoardo Bassini 15, 20133 Milano, Italy
}

Received 10 August 2008 / Accepted 10 January 2009

\section{ABSTRACT}

\begin{abstract}
Aims. XTE J1810-197 is the first transient anomalous X-ray pulsar ever discovered. Its highly variable X-ray flux allowed us to study the timing and spectral emission properties of a magnetar candidate over a flux range of about two orders of magnitude.

Methods. We analyzed nine XMM-Newton observations of XTE J1810-197 collected over a four year baseline (September 2003September 2007). EPIC PN and MOS data were reduced and used for detailed timing and spectral analysis. Pulse-phase spectroscopic studies were also carried out for observations with a high enough signal-to-noise.

Results. We find that (i) a three-blackbody model reproduces the spectral properties of XTE J1810-197 over the entire outburst statistically better than the two blackbodies model previously used in the literature, (ii) the coldest blackbody is consistent with the thermal emission from the whole surface and has temperature and radius similar to those inferred from ROSAT observations before the outburst onset, (iii) there is a spectral feature around $1.1 \mathrm{keV}$ during six consecutive observations (since March 2005). If this stems from proton resonant cyclotron scattering, it would imply a magnetic field of $\sim 2 \times 10^{14} \mathrm{G}$. This closely agrees with the value from the spin period measurements
\end{abstract}

Key words. moon - stars: magnetic fields - stars: neutron - X-rays: stars

\section{Introduction}

Even though isolated neutron stars as a whole are relatively poor X-ray emitters, two small classes of objects stand out for their widely variable high-energy emission, which covers several orders of magnitude both in intensity and in timescales. These objects are the anomalous X-ray pulsars (AXPs; ten objects plus one candidate) and soft $\gamma$-ray repeaters (SGRs; 5 objects plus 2 candidates; for a review see Woods et al. 2006). It is believed that AXPs and SGRs are linked at some level, owing to their similar timing properties (spin periods in the 2-12 s range and period derivatives $\dot{P}$ in the $10^{-13} \div 10^{-11} \mathrm{~s} \mathrm{~s}^{-1}$ range). Both classes have been proposed to consist of neutron stars whose emission is powered by the decay of their extremely strong internal magnetic fields ( $>10^{15} \mathrm{G}$; Duncan \& Thompson 1992; Thompson \& Duncan 1995). Different types of X-ray flux variability are displayed by AXPs. From slow and moderate flux changes (up to a factor of a few) on timescales of years (virtually all of the objects of the class), to moderate-intense outbursts (flux variations of a factor up to 10) lasting for 1-3 years (1E 2259+586, and 1E 1048.1-5973), to dramatic and intense SGR-like burst activity (fluences of $10^{36}-10^{38} \mathrm{erg}$ ) on subsecond timescales (4U 0142+614, XTE J1810-197, 1E 2259+586, and
1E 1048.1-5973; see for a review of the X-ray variability see Kaspi et al. 2007). The first notable recorded case of flux variability was the 2002 bursting/outbursting event detected from $1 \mathrm{E} 2259+586$, in which a factor of $\sim 10$ persistent flux enhancement in an AXP was followed by the onset of bursting activity during which the source emitted more than 80 short bursts (Gavriil et al. 2004; Woods et al. 2004). The timing and spectral properties of the source changed significantly and attained the pre-bursting activity values within a few days.

However, it was only in 2003 that the first transient AXP (TAXP), namely XTE J1810-197, was discovered (Ibrahim et al. 2004). This source was serendipitously detected by the $R X T E$ satellite, and then localized and studied in greater detail with the Chandra and XMM-Newton observatories (Gotthelf et al. 2004; Israel et al. 2004; Rea et al. 2004; Gotthelf \& Halpern 2005, 2007). It displayed a persistent flux enhancement by a factor of $>100$ with respect to the quiescent luminosity level of $\sim 10^{33} \mathrm{erg} \mathrm{s}^{-1}$ (as observed by ROSAT and Einstein observatories). Unfortunately, the initial phases of the outburst were missed so we do not know whether a bursting phase, similar to that of $1 \mathrm{E} 2259+586$, also occurred for this source soon after the onset of the outburst. However, four bursts were detected by RXTE between September 2003 and April 2004 and 
unambiguously associated with XTEJ1810-197 (Woods et al. 2005). By using Very Large Array (VLA) archival data, Helfand et al. (2005) discovered a transient radio emission with a flux of $\sim 4.5 \pm 0.5 \mathrm{mJy}$ at $1.4 \mathrm{GHz}$ at the Chandra X-ray position of XTE J1810-197. Only later its emission was discovered to be pulsed, highly polarized and to have large flux variability even on very small timescales (at variance with all known radio pulsars; Camilo et al. 2006). The VLA data were also used to infer a proper motion of $13.5 \pm 1.0 \mathrm{mas} \mathrm{yr}^{-1}$, which, assuming a distance of $3.5 \pm 0.5 \mathrm{kpc}$, results in a transverse velocity of $212 \pm 35 \mathrm{~km} \mathrm{~s}^{-1}$ ( $1 \sigma$ confidence level; Helfand et al. 2007).

Deep IR observations have revealed a weak, $K_{\mathrm{s}}=20.8 \mathrm{mag}$ counterpart, with characteristics similar to those of other AXPs (Israel et al. 2004). Variability in the IR counterpart of XTE J1810-197 was found (Rea et al. 2004), but it did not correlate with the X-ray emission, contrary to earlier claims (Camilo et al. 2007a; Testa et al. 2008). It is unclear at present whether the IR variability correlates with what is observed in the radio pulsed emission (Camilo et al. 2006, 2007a).

TAXP are fairly rare objects: a second TAXP was revealed in 2006 when a candidate AXP, namely CXOU J164710.2-455216, displayed a rather intense burst followed by an outburst with a maximum flux enanchement $>300$, characterized by extreme changes in both the spectral and timing properties (Muno et al. 2006a,b; Israel et al. 2007a). At variance with XTE J1810-197, CXOU J164710.2-455216 has not shown any radio emission so far. The third TAXP, 1E1547.0-5408, was discovered in 2007 when its X-ray flux rose by a factor of $\sim 20$ above the quiescent flux. As in the case of XTE J1810-197, 1E1547.0-5408 was found to be a transient radio pulsar. Unfortunately, the observations missed the outburst onset (Camilo et al. 2007b; Gelfand \& Gaensler 2007; Halpern et al. 2008). The three TAXPs above are characterized by a quiescent state, the timing and spectral properties of which are similar to those of thousands of other $\mathrm{X}$-ray sources present in the ROSAT catalog: no pulsations (with the exception of CXOU J164710.2-455216) and soft X-ray spectra well-fitted by a blackbody $(B B)$ model with a $k T$ of about 0.1-0.2 keV; again with the exception of CXOUJ164710.2455216, which has $k T \sim 0.5 \mathrm{keV}$ (Muno et al. 2006b; Skinner et al. 2006). The transient nature of these three AXPs implies that a relatively large number of members of this class has not been discovered yet and suggests that others will manifest themselves in the future through their outbursts.

After more than four years of data since the outburst onset, XTEJ1810-197 provides the first opportunity to study the timing and spectral evolution of a TAXP as it returns to its quiescent state. Since the first XMM-Newton 2003 observations of XTEJ1810-197 (Gotthelf et al. 2004), carried out approximately one year after the outburst, it was evident that the source spectrum (two blackbodies with $k T_{1}=0.29 \pm 0.03 \mathrm{keV}$, $R_{\mathrm{BB} 1} \sim 5.5 \mathrm{~km}$, and $k T_{2}=0.70 \pm 0.02 \mathrm{keV}, R_{\mathrm{BB} 2} \sim 1.5 \mathrm{~km}$; $L_{\mathrm{X}} \sim 5 \times 10^{34} \mathrm{erg} \mathrm{s}^{-1}$ in the $0.5-10 \mathrm{keV}$ range for a distance of $3.5 \mathrm{kpc}$ ) was significantly different from that in quiescence recorded by ROSAT in 1992 (one $B B$ with $k T \approx 0.18 \mathrm{keV}$ and $R_{\mathrm{BB}} \approx 10 \mathrm{~km}$; extrapolated luminosity in the $0.5-10 \mathrm{keV}$ range of $L_{\mathrm{X}} \sim 7 \times 10^{32} \mathrm{erg} \mathrm{s}^{-1}$; Gotthelf et al. 2004). Moreover, the source showed $5.54 \mathrm{~s}$ pulsations with a pulsed fraction of about $45 \%$ during outburst, while only an upper limit $24 \%$ was inferred from the ROSAT data (Gotthelf et al. 2004).

The above properties raise a number of important, still unanswered questions. Is the soft $B B$ component detected by $X M M$-Newton evolving into the quiescent $B B$ component seen by ROSAT? What happens to the higher temperature $B B$ component as the source approaches quiescence? What is the pulsed fraction level of the source in quiescence (if detectable)? Is the quiescent emission revealing the neutron star (NS) cooling surface? Did the outburst lead to a permanent change of the timing/spectral properties, such as the pulsed fraction, the flux, and temperature or size of the quiescent $B B$ component of the source? What is the intensity of the magnetic field of this source?

In this paper, we present a first attempt to answer the above questions through a detailed study of the timing and spectral evolution of XTE J1810-197 during its outburst decay in 2003-2007. In Sect. 2 we report the details of the XMM-Newton observations and our data analysis strategy. Results are presented in Sect. 3, while their implications are discussed in Sect. 4.

\section{Observations and data analysis}

XTEJ1810-197 was observed with XMM-Newton at nine epochs, the first time for just $\sim 5 \mathrm{ks}$, while the remaining eight observations were deeper, from $\sim 11 \mathrm{ks}$ to $\sim 60 \mathrm{ks}$ (Table 1). The XMM-Newton Observatory (Jansen et al. 2001) includes three $\sim 1500 \mathrm{~cm}^{2}$ X-ray telescopes with an EPIC instrument in each focus, a Reflecting Grating Spectrometer (RGS; den Herder et al. 2001) and an Optical Monitor (Mason et al. 2001). Two of the EPIC imaging spectrometers use MOS CCDs (Turner et al. 2001) and one uses a PN CCD (Strüder et al. 2001). Data were processed with SAS version 7.1.0, using the updated calibration files (CCF) available in June 2008. Standard data screening criteria are applied in the extraction of scientific products. We cleaned all observations from solar flares by collecting CCD lightcurves above $10 \mathrm{keV}$ and applying an intensity threshold. We also used a time window criterion for removing solar flare intervals and checked that no significant spectral differences were present with respect to the intensity threshold method.

During the September 2003 observation, the PN camera was set in primary small window imaging mode with a thin filter (time resolution $=5.07 \times 10^{-3} \mathrm{~s}$ ), while all other observations were in a primary large window imaging mode with a medium filter (time resultion $=4.76 \times 10^{-2} \mathrm{~s}$ ). All observation set-ups for MOS1 and MOS2 cameras were the same, with a time resolution of $0.3 \mathrm{~s}$ : prime partial window imaging mode and medium filter. In September 2003 the MOS1 was set in prime full window imaging mode, in September 2004 the MOS2 was in Timing uncompressed mode and data from this were not reduced. To extract more than $90 \%$ of the source counts, we accumulated a one-dimensional image and fitted the $1 \mathrm{D}$ photon distribution with a Gaussian. Then, we extracted the source photons from a circular region of radius $55^{\prime \prime}$ ( $~ 90 \%$ of photons) centered at the Gaussian centroid. The background for the spectral analysis was obtained (within the same PN or MOS CCD where the source lies) from an annulus region (inner and outer radii of $65^{\prime \prime}$ and $100^{\prime \prime}$, respectively) centered at the best source position. In the timing analysis, the background was estimated from a circular region of the same size as that of the source. All of the EPIC spectra were rebinned before fitting, to have at least 40 counts per bin and prevent oversampling the energy resolution by more than a factor of three. Thanks to the time and spectral resolution of the EPIC instruments ${ }^{1}$, we could carry out timing and spectral analysis over the entire set of observations and the pulse phase spectroscopy (PPS) for the observations with a high enough signal-to-noise. We report the analyses obtained with the PN data and, for comparison, the results from the two MOS cameras.

\footnotetext{
1 http://xmm.esac.esa.int/external/xmm_user_ support/..... documentation/uhb_2.5/node28.html
} 
Table 1. Main observational parameters for the nine XMM-Newton datasets using for the uncertainties a $1 \sigma$ confidence level.

\begin{tabular}{|c|c|c|c|c|c|c|c|}
\hline Epoch & $\begin{array}{l}\text { Period } \\
\mathrm{s}\end{array}$ & Instrum. (mode) & $\begin{array}{l}\text { Exp. Time } \\
\text { S }\end{array}$ & tot ph-bck ph & $\tau_{\max }$ edge & $\begin{array}{c}\text { Energy edge } \\
\mathrm{keV}\end{array}$ & $\chi_{\text {red }}^{2}$ \\
\hline \multirow[t]{3}{*}{ Sep. 2003} & $5.53928(3)$ & EPN & 5199 & $60136-2903$ & $<0.17$ & 1.10(fix) & a \\
\hline & & MOS1 & 7700 & $30761-111$ & $<0.02$ & 1.10 (fix) & 1.33 \\
\hline & & MOS2 & 7800 & 26 739-145 & $<0.03$ & 1.10 (fix) & \\
\hline \multirow[t]{3}{*}{ Mar. 2004} & $5.53945(1)$ & EPN & 10730 & 71 180-3077 & $<0.18$ & 1.10 (fix) & \\
\hline & & MOS1 & 12000 & $27932-396$ & $<0.03$ & 1.10 (fix) & 1.15 \\
\hline & & MOS2 & 12200 & $28809-366$ & $<0.05$ & 1.10 (fix) & \\
\hline \multirow[t]{3}{*}{ Sep. 2004} & $5.539599(6)$ & EPN & 21306 & $89082-1574$ & $<0.17$ & 1.10(fix) & \\
\hline & & MOS1 & 24000 & 35 515-263 & $<0.06$ & 1.10 (fix) & 1.22 \\
\hline & & MOS2 & timing mode & timing mode & timing mode & timing mode & timing mode \\
\hline \multirow[t]{3}{*}{ Mar. 2005} & $5.539825(6)$ & EPN & 24988 & $54279-1760$ & $0.12 \pm 0.03$ & $1.14 \pm 0.02$ & \\
\hline & & MOS1 & 37800 & $26501-428$ & $0.09 \pm 0.04$ & $1.10 \pm 0.03$ & 1.05 \\
\hline & & MOS2 & 37800 & $28004-330$ & $0.10 \pm 0.02$ & $0.98 \pm 0.08$ & 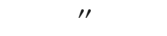 \\
\hline \multirow[t]{3}{*}{ Sep. 2005} & $5.54004(1)$ & EPN & 19787 & $21876-1311$ & $0.26 \pm 0.04$ & $1.07 \pm 0.02$ & \\
\hline & & MOS1 & 30000 & $10562-344$ & $0.2 \pm 0.1$ & $1.13 \pm 0.03$ & 1.05 \\
\hline & & MOS2 & 18000 & $6645-146$ & $<0.4$ & 1.10 (fix) & " \\
\hline \multirow[t]{3}{*}{ Mar. 2006} & $5.54022(3)$ & EPN & 15506 & 12296-1197 & $0.17 \pm 0.05$ & $1.11 \pm 0.03$ & \\
\hline & & MOS1 & 26500 & 6539-338 & $0.3 \pm 0.3$ & $1.48 \pm 0.06$ & 0.95 \\
\hline & & MOS2 & 28000 & $7119-328$ & $0.2 \pm 0.1$ & $1.02 \pm 0.02$ & 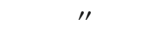 \\
\hline \multirow[t]{3}{*}{ Sep. 2006} & $5.54037(1)$ & EPN & 38505 & $23842-2974$ & $0.13 \pm 0.03$ & $1.07 \pm 0.02$ & \\
\hline & & MOS1 & 46800 & $8113-552$ & $0.20 \pm 0.05$ & $1.02 \pm 0.02$ & 1.47 \\
\hline & & MOS2 & 46500 & $8836-558$ & $0.08 \pm 0.06$ & $0.93 \pm 0.03$ & $"$ \\
\hline \multirow[t]{3}{*}{ Mar. 2007} & $5.54041(1)$ & EPN & 37296 & $21903-2215$ & $0.21 \pm 0.04$ & $1.07 \pm 0.02$ & \\
\hline & & MOS1 & 63000 & 4410-1897 & $0.14 \pm 0.04$ & $0.93 \pm 0.02$ & 0.99 \\
\hline & & MOS2 & 53000 & $4635-522$ & $0.15 \pm 0.05$ & $1.10 \pm 0.02$ & " \\
\hline \multirow[t]{3}{*}{ Sep. 2007} & $5.540472(7)$ & EPN & 59014 & $34386-4117$ & $0.18 \pm 0.02$ & $1.04 \pm 0.06$ & \\
\hline & & MOS1 & 67910 & $11038-752$ & $0.17 \pm 0.04$ & $1.09 \pm 0.02$ & 1.26 \\
\hline & & MOS2 & 68785 & $12328-768$ & $0.10 \pm 0.03$ & $1.1 \pm 0.1$ & " \\
\hline
\end{tabular}

${ }^{a}$ The nine PN spectra were fitted together according to the prescription discussed in the text (3.2.3); the resulting reduced $\chi^{2}\left(\chi_{\text {red }}^{2}\right)$ is 1.09 (1038 d.o.f.), for the $3 B B+$ edge model.

\section{Results}

\subsection{Timing analysis}

The source event arrival time of each observation, in the $0.5-15 \mathrm{keV}$ energy range, were converted into barycentric dynamical times (BDT) by means of the SAS tool barycen and the $\left(\sim 1^{\prime \prime}\right.$ accurate) source position provided by Helfand et al. (2007). Given the complex time evolution of the period and its derivatives from radio observations (Camilo et al. 2007c), we measured only the local spin period at each single XMM-Newton pointing by means of a phase-fitting technique (events in the $0.5-10 \mathrm{keV}$ energy range were used; see e.g. Dall'Osso et al. 2003, for details on the technique). Different period measurements are independent and not phase-connected. Folding each lightcurve at its measured spin period we obtained the pulse profile and found that it remained single-peaked in all observations (Fig. 1). To estimate the dipole field strength of this source we refer to the phase-coherent measurements of $v, \dot{v}$, and $\ddot{v}$ obtained by Camilo et al. (2007c). These authors measured fast variations of $\dot{v}$ that did not allow them to provide a unique value for the magnetic field strength. The frequency derivative was found to change continuously over 300 days of monitoring from $\sim-3.4 \times 10^{-13} \mathrm{~s}^{-2}$ to $\sim-1.4 \times 10^{-13} \mathrm{~s}^{-2}$. Accordingly, we considered the secular spindown trend as bracketed by these limits and derived a corresponding range of values for the magnetic field $1.6 \times 10^{14} \mathrm{G} \leq B_{\text {dip }} \leq 2.8 \times 10^{14} \mathrm{G}$ through the standard dipole formula.

\subsubsection{Pulsed fraction}

Given the smooth and nearly sinusoidal pulse shape, we could determine the pulsed fraction $(\mathrm{PF})$ of the signal (defined here as: $\mathrm{PF}=\left(A_{\max }-A_{\min }\right) /\left(A_{\max }+A_{\min }\right)$ with reasonable accuracy, where $A_{\max }$ and $A_{\min }$ are the maximum and minimum of the sinusoidal modulation). Between September 2003 and September 2007 , the PF decreased by a factor of about two (between $\sim 50 \%$ and $\sim 25 \%$ ) in the $0.5-10 \mathrm{keV}$ energy interval (Fig. 2). In particular, since March 2005, the PF in the $0.1-2.5 \mathrm{keV}$ band has reached $\sim(25 \pm 1) \%$ (here and troughout this paper uncertainties are given at $1 \sigma$ confidence level, where not stated otherwise); this value is close to the upper limit $(\sim 24 \%)$ inferred from the 1992-1993 ROSAT pointings during the quiescent phase of the source (Gotthelf et al. 2004).

Moreover, the PF decreases as a function of time in the same energy band and increases as a function of energy within the same observation, as shown in Table 2. Between 8 and $15 \mathrm{keV}$, the pulsed fraction is consistent with $100 \%$ ( $3 \sigma$ confidence level). However, the relatively poor statistics above $10 \mathrm{keV}$ prevented a detailed study of the spectral properties of this highenergy component (see also Sect. 3.2).

\subsection{Spectral analysis}

In the following we describe a detailed spectral analysis of our XMM-Newton dataset, which includes the outburst evolution down to its almost complete decay. The outburst spectrum in its brightest phase had already been analyzed in the 


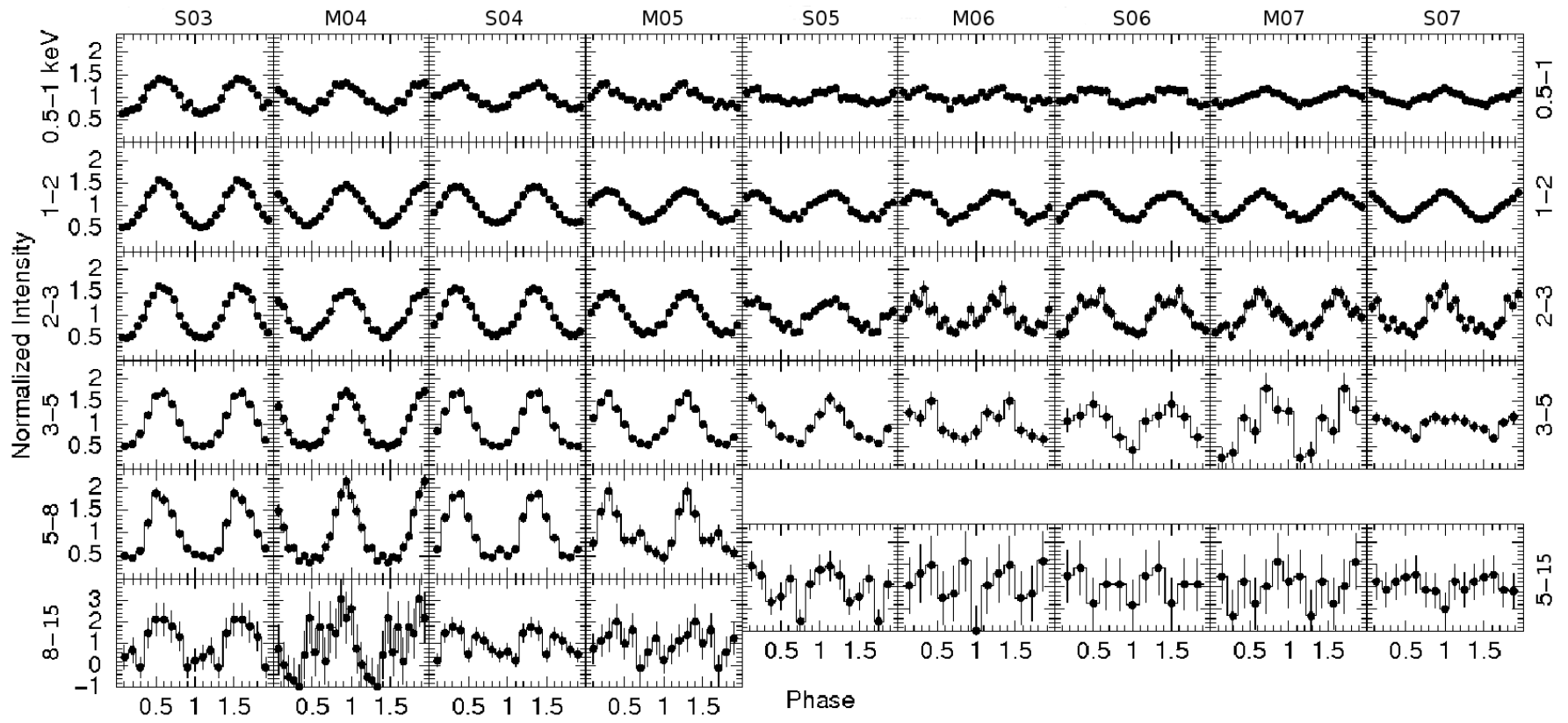

Fig. 1. XTE J1810-197 PN background-subtracted lightcurves folded at the best period (see Table 1) for each of the nine XMM-Newton observations carried out between September 2003 (S03) and September 2007 (S07), and for different energy intervals: 0.5-1 keV, 1-2 keV, 2-3 keV, 3-5 keV, 5-8 keV, and 8-15 keV. The last two energy intervals have been merged together since the September 2005 (S05) pointing in order to improve the statistics.
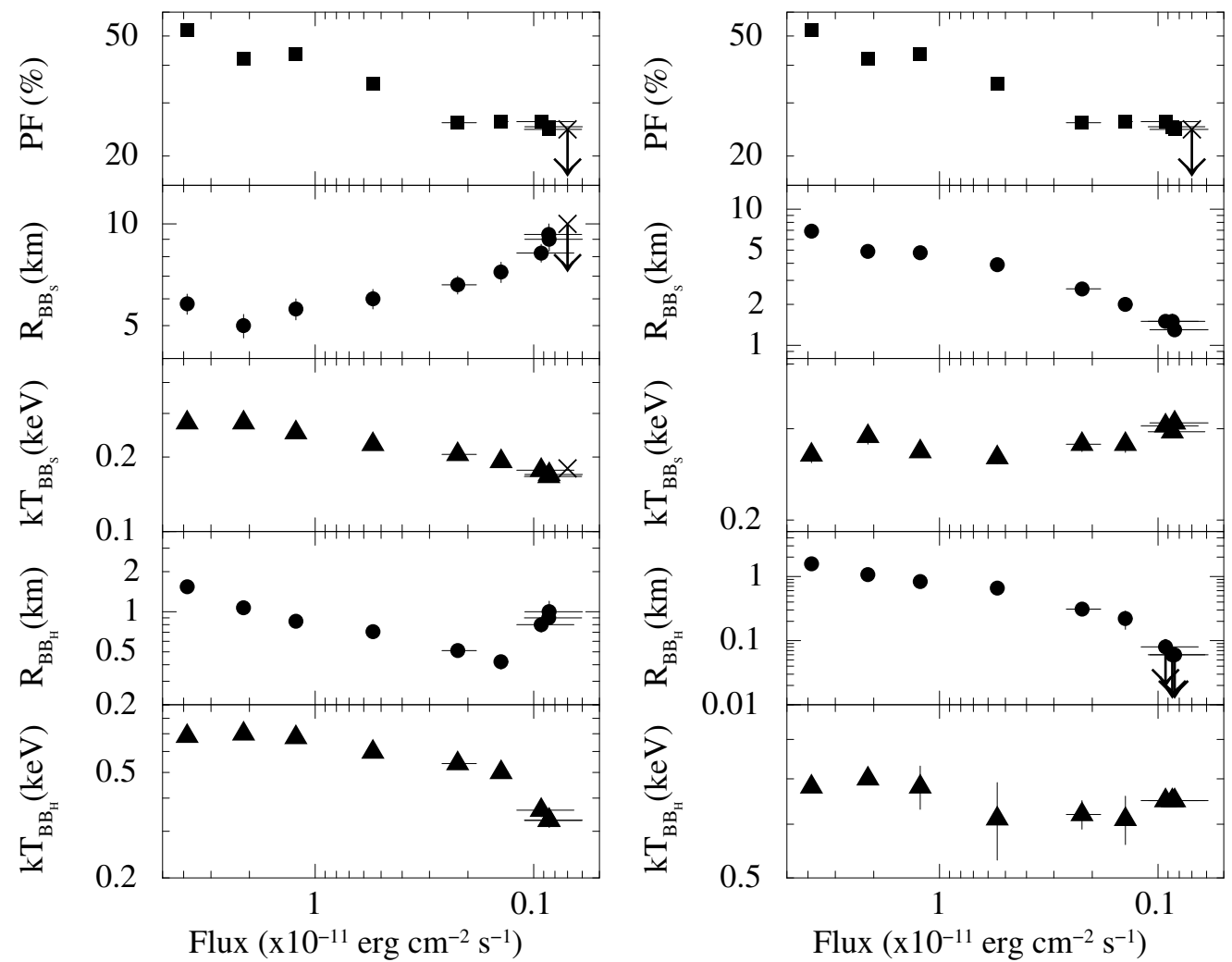

Fig. 2. Evolution of the spectral parameters for the $2 B B$ (left panel) and $3 B B$ (right panel) models together with the pulsed fraction (PF) as a function of the 0.6-10 keV flux. The cross in the left panel (first, second, and third rows), and in the right panel (first row) marks the ROSAT data.

literature with a two-blackbody spectral model $(2 B B$, Gotthelf et al. 2004; Gotthelf \& Halpern 2005). On the other hand, the quiescent emission from the source recorded by ROSAT was consistent with a different, single $B B$. Starting from this, our strategy was then twofold: first we tried to apply the $2 B B$ model to the whole XMM-Newton dataset to check whether one of the two components evolved smoothly to the quiescent one. Then we tested an alternative possibility, namely that the quiescent component was independent and always present, the spectrum of the outburst being superimposed on it. This led us to consider a 
Table 2. Pulsed fraction (PF) in different energy intervals vs. time, and $P F_{0.5 \div 15}^{\text {total }} \mathrm{keV}$. Errors are reported at $1 \sigma$ confidence level.

\begin{tabular}{cccccccc}
\hline \hline Epoch & $\mathrm{PF}_{0.5 \div 1 \mathrm{keV}}$ & $\mathrm{PF}_{1 \div 2 \mathrm{keV}}$ & $\mathrm{PF}_{2 \div 3 \mathrm{keV}}$ & $\mathrm{PF}_{3 \div 5 \mathrm{keV}}$ & $\mathrm{PF}_{5 \div 8 \mathrm{keV}}$ & $\mathrm{PF}_{8 \div 15 \mathrm{keV}}$ & $\mathrm{PF}_{0.5 \div 15 \mathrm{keV}}^{\text {total }}$ \\
& $\%$ & $\%$ & $\%$ & $\%$ & $\%$ & $\%$ & $\%$ \\
\hline Sep. 2003 & $35 \pm 2$ & $49.3 \pm 0.5$ & $57.0 \pm 0.8$ & $58.7 \pm 0.9$ & $63 \pm 3$ & $100 \pm 20$ & $52.4 \pm 0.4$ \\
Mar. 2004 & $25 \pm 1$ & $37.2 \pm 0.5$ & $42.1 \pm 0.8$ & $51 \pm 1$ & $66 \pm 3$ & $50 \pm 25$ & $42.0 \pm 0.4$ \\
Sep. 2004 & $27 \pm 1$ & $40.2 \pm 0.7$ & $49.6 \pm 0.7$ & $57.6 \pm 0.9$ & $63 \pm 2$ & $50 \pm 12$ & $43.5 \pm 0.3$ \\
Mar. 2005 & $20 \pm 1$ & $33.1 \pm 0.6$ & $44 \pm 1$ & $51 \pm 1$ & $49 \pm 5$ & $100 \pm 40$ & $34.8 \pm 0.4$ \\
Sep. 2005 & $13 \pm 2$ & $27.2 \pm 0.9$ & $32 \pm 2$ & $30.0 \pm 0.9$ & $60 \pm 11$ & $60 \pm 30$ & $25.8 \pm 0.7$ \\
Mar. 2006 & $13 \pm 2$ & $30 \pm 1$ & $32 \pm 3$ & $26 \pm 6$ & $70 \pm 29$ & $20 \pm 48$ & $26 \pm 1$ \\
Sep. 2006 & $16 \pm 1$ & $29.3 \pm 0.9$ & $42 \pm 3$ & $90 \pm 72$ & $90 \pm 72$ & $40 \pm 41$ & $26 \pm 0.7$ \\
Mar. 2007 & $15 \pm 2$ & $32 \pm 1$ & $36 \pm 3$ & $60 \pm 10$ & $30 \pm 33$ & $40 \pm 10$ & $25 \pm 1$ \\
Sep. 2007 & $15 \pm 1$ & $28.1 \pm 0.7$ & $41 \pm 3$ & $50 \pm 10$ & $40 \pm 50$ & $80 \pm 25$ & $24.5 \pm 0.6$ \\
\hline
\end{tabular}

phenomenological model including three different thermal components ( $3 B B$ model). In this scenario, as the outburst flux decays, its spectral components progressively fade away, eventually revealing the underlying quiescent emission. As such, the quiescent component could be tentatively identified with the thermal emission from the whole NS surface.

\subsubsection{Thermal components}

Following Gotthelf et al. (2004) and Gotthelf \& Halpern (2005), we applied the $2 B B$ spectral fit to the fading phases of XTE J1810-197 until September 2007, when the source flux was $\sim 1.2$ times higher than the pre-outburst level $\left(\chi_{\text {red }}^{2} \sim 1.26\right.$ for 1058 d.o.f., which is at $6 \sigma_{\chi^{2}}$ from the expectation value ${ }^{2}$; $N_{\mathrm{H}}=(0.60 \pm 0.01) \times 10^{22} \mathrm{~cm}^{-2}$; see also Table 3$)$. The $2 B B$ model (see also Perna \& Gotthelf 2008, for a detailed study) corresponds to a scenario in which one of the two $B B$ naturally evolves into the single $B B$ spectrum detected by ROSAT in the quiescent state, whereas the other (hard) $B B$ just fades away. The results of this approach showed that, when the cold $B B$ component smoothly approaches the quiescent one (see Fig. 2, left panel, 2nd and 3rd plot), several ambiguities arise. The radius of the hot $B B$ does not decrease monotonically with flux (time): after 2.5 years of smooth decrease it starts increasing in September 2006 (left panel, 4th plot). At the same epoch its temperature drops rapidly to reach a value comparable to that of the cold $B B$ in the first part of the outburst (Fig. 2, left panel, 5th plot). Moreover, neither spectral component is able to account for the flattening of the pulsed fraction at the $25 \%$ level (Fig. 2, left panel, 1st plot).

These findings suggest that the observed emission might come from a more complex structure than a simple twocomponent model and that we might be seeing different parts of the whole structure as the flux decreases. With this scenario in mind we repeated the spectral analysis using the $3 B B$ model discussed in the previous section.

For the first six observations (during which the total flux is significantly higher than the pre-outburst one), all parameters of the $3 B B$ model were left free to vary except for $N_{\mathrm{H}}$, which was constrained to be the same in all observations. We found that it is always possible to fit the first 6 data sets (September 2003-March 2006) with a $3 B B$ model without forcing the spectral parameters $\left(3 B B: \chi_{\text {red }}^{2}=1.1,812\right.$ d.o.f.; $2 B B$ : $\chi_{\text {red }}^{2}=1.21,824$ d.o.f., F-test probability $\simeq 10^{-11} \sim 7 \sigma$ ). The extra $B B$ has a characteristic temperature $k T \sim 0.14 \mathrm{keV}$ that is

${ }^{2} \sigma_{\chi^{2}}^{2}=2$ d.o.f. $\Rightarrow \sigma_{\chi^{2}}=\sqrt{2 \text { d.o.f., }} \frac{\left(\chi^{2} \text {-d.of. }\right)}{\sigma_{\chi^{2}}}=x\left[\sigma_{\chi^{2}}\right]$, where $x$ is the distance from the expectation value of $\chi^{2}$ in units of $\sigma_{\chi^{2}}$.
Table 3. Temperature $(\mathrm{keV})$ and radius $(\mathrm{km})$ evolution with time of $B B_{\text {cold }}$ and $B B_{\text {hot }}$ in the $2 B B$ model, with uncertainties at $1 \sigma$ confidence level $(68 \%)$.

\begin{tabular}{ccccc}
\hline \hline Epoch & $\begin{array}{c}k T_{\text {cold }} \\
\mathrm{keV}\end{array}$ & $\begin{array}{c}R_{\text {cold }} \\
\mathrm{km}\end{array}$ & $\begin{array}{c}k T_{\text {hot }} \\
\mathrm{keV}\end{array}$ & $\begin{array}{c}R_{\text {hot }} \\
\mathrm{km}\end{array}$ \\
\hline Sep. 03 & $0.275 \pm 0.009$ & $5.8 \pm 0.4$ & $0.685 \pm 0.005$ & $1.54 \pm 0.03$ \\
Mar. 04 & $0.275 \pm 0.009$ & $5.0 \pm 0.4$ & $0.699 \pm 0.009$ & $1.07 \pm 0.03$ \\
Sep. 04 & $0.251 \pm 0.005$ & $5.6 \pm 0.4$ & $0.677 \pm 0.005$ & $0.85 \pm 0.02$ \\
Mar. 05 & $0.225 \pm 0.004$ & $6.0 \pm 0.4$ & $0.597 \pm 0.006$ & $0.71 \pm 0.02$ \\
Sep. 05 & $0.205 \pm 0.004$ & $6.6 \pm 0.4$ & $0.54 \pm 0.01$ & $0.51 \pm 0.04$ \\
Mar. 06 & $0.192 \pm 0.004$ & $7.2 \pm 0.5$ & $0.50 \pm 0.02$ & $0.42 \pm 0.05$ \\
Sep. 06 & $0.177 \pm 0.004$ & $8.2 \pm 0.5$ & $0.36 \pm 0.02$ & $0.8 \pm 0.1$ \\
Mar. 07 & $0.170 \pm 0.005$ & $9.0 \pm 0.7$ & $0.33 \pm 0.02$ & $1.0 \pm 0.2$ \\
Sep. 07 & $0.167 \pm 0.004$ & $9.3 \pm 0.7$ & $0.33 \pm 0.01$ & $0.9 \pm 0.1$ \\
\hline
\end{tabular}

Given the stability of both the flux and spectrum over the last three observations (less than $8 \%$ variation in flux in the $0.6-10 \mathrm{keV}$ energy band; September 2006-2007), we merged the source photon lists to obtain a higher $\mathrm{S} / \mathrm{N}$ spectrum. The calibration of the $\mathrm{PN}$ instrument has proved to be also very stable (Krisch et al. 2005). The analysis of the merged spectrum significantly improved the determination of the spectral parameters as compared to each single spectrum (see Table 4). Furthermore, the hotter $B B$ component remained statistically non-significant also in the merged spectrum. We could thus obtain a more accurate $(3 \sigma)$ upper limit on its flux: $F_{\text {BBhot }}<4.5 \times 10^{-14} \mathrm{erg} \mathrm{cm}^{-2} \mathrm{~s}^{-1}$ in the $0.6-10 \mathrm{keV}$ energy band.

constant in time, but whose radius could not be well-constrained $(R<100 \mathrm{~km})$. Under the hypothesis that the latter component originates in the whole NS surface, we can consider it constant through the whole outburst. Correspondingly, we left the temperature and radius of this additional $B B$ free to vary, but forced both parameters to maintain the same value in all spectra.

We then applied the $3 B B$ model to all of the $9 X M M$-Newton observations. The addition of the extra $B B$ component gave a better fit than the $2 B B$ model $\left(\chi^{2} \sim 1250, \chi_{\text {red }}^{2} \sim 1.18\right.$ for 1056 d.o.f., $\left.N_{\mathrm{H}}=(0.72 \pm 0.02) \times 10^{22} \mathrm{~cm}^{-2}\right)$; an F-test gives a $7.3 \sigma$ significance for the inclusion of the additional spectral component. Notably, the overall fit gave parameters for the coldest $B B, k T_{\text {cold }}=0.144 \pm 0.003 \mathrm{keV} ; R_{\text {cold }}=17.9 \pm \pm_{1.5}^{1.9} \mathrm{~km}$, $F_{\mathrm{X}}^{0.1-2.5 \mathrm{kev}}=(4.5 \pm 0.5) \times 10^{-13} \mathrm{erg} \mathrm{cm}^{-2} \mathrm{~s}^{-1}$ which are very close to those inferred in quiescence with ROSAT $(k T=0.18 \pm$ $0.02 \mathrm{keV}$ and $R \sim 10 \mathrm{~km}, F_{\mathrm{X}}^{0.1-2.5} \sim 5.4 \times 10^{-13} \mathrm{erg} \mathrm{cm}^{-2} \mathrm{~s}^{-1}$ ). Even more interesting, the two hotter $B B$ components maintained a nearly constant temperature as the source flux decayed in time (see Fig. 2, right panel, 3rd ad 5th plots). Their radius appears to be the only variable parameter during the decaying phase of the outburst (Fig. 2, right panel, 2nd and 4th plots).

Starting from September 2006 the spectrum could be wellfitted by a simple $2 B B$ model. The hottest component was not 


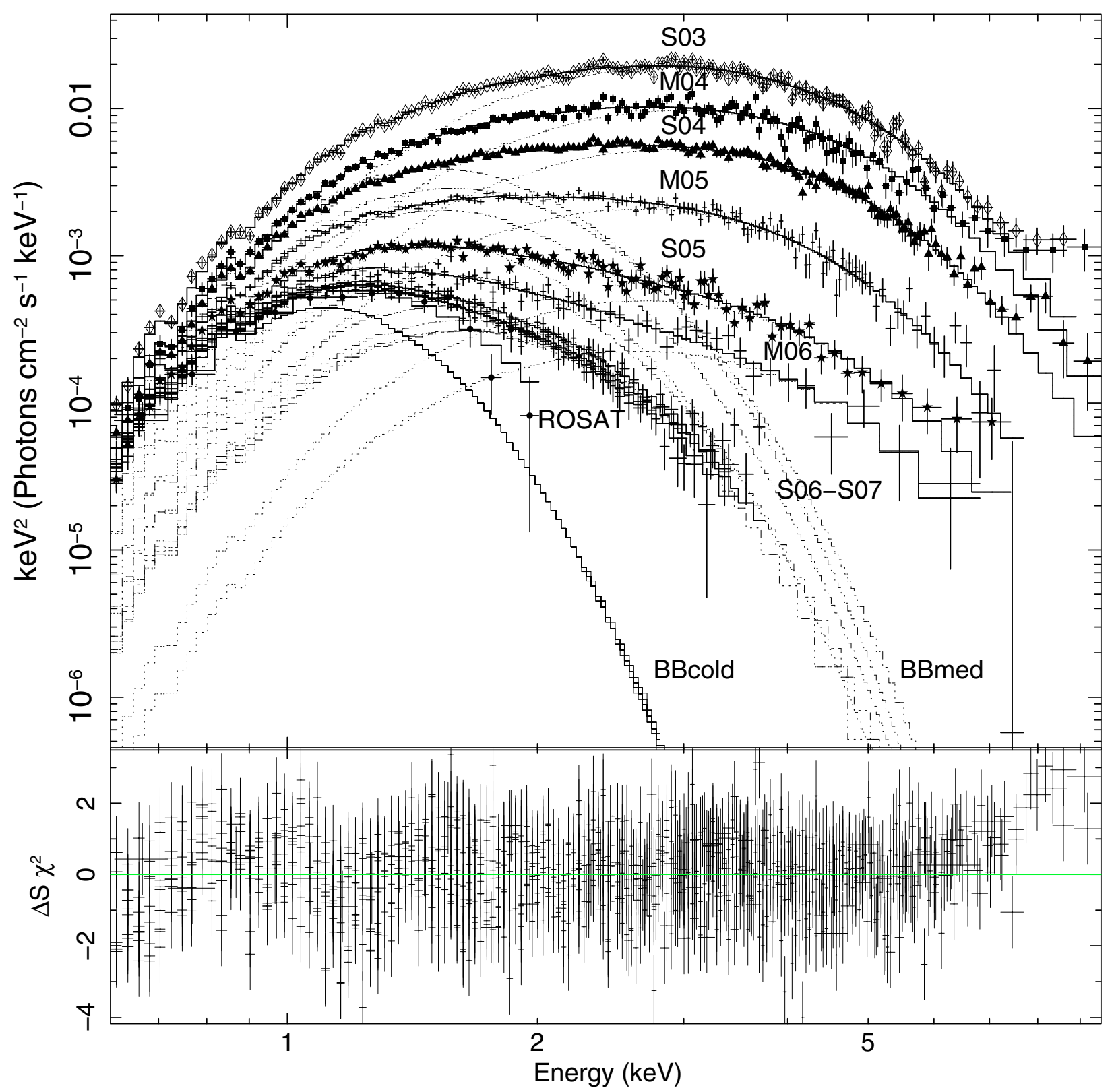

Fig. 3. Spectral evolution in the nine XMM-Newton observations, and the ROSAT spectrum in quiescence. Fits use the $3 B B$ model (upper panel). Model residuals are shown in the lower panel. The soft component, constant in time, is marked with $B B_{\text {cold }}$; the medium-temperature evolving blackbody component is marked with $B B_{\text {med. }}$ There is a sharp drop in the flux above $2.5 \mathrm{keV}$ in the last three spectra (compare M06 with S06, M07, and S07) which corresponds to the disappearance of the hot component $B B_{\text {hot }}$. M = March, S = September; $03=2003,04=2004,05=2005$, $06=2006$, and $07=2007$.

needed anymore and we could set a $3 \sigma$ upper limit on its flux of $\sim 8.7 \times 10^{-14} \mathrm{erg} \mathrm{cm}^{-2} \mathrm{~s}^{-1}$. The above-mentioned flattening of the pulsed fraction at this epoch could be accounted for quite naturally by the disappearance of this hot component (Fig. 3).

We also attempted to estimate the pulsed fraction of the quiescent emission, which we tentatively attributed to the NS surface, taking only the last three observations into account, so that the hotter $B B$ was absent. We note that this is only possible in our scenario since the softer component in the $2 B B$ model is still evolving towards quiescence.

We express the PF in the $0.1-1 \mathrm{keV}$ band as $\mathrm{PF}_{(0.1-1 \mathrm{keV})}=$ $\alpha F_{\text {cold }}+\beta F_{\text {med }}$, where $F_{\text {cold }} \sim 0.9$ and $F_{\text {med }} \sim 0.1$ represent the relative contributions of the two spectral components to the total flux in the $0.1-1 \mathrm{keV}$ band. Correspondingly, $\alpha$ and $\beta$ represent their PFs. The value of $\beta$ is obtained from the PF in the $2.8-4.1 \mathrm{keV}$ energy range, where $B B_{\text {cold }}$ is negligible, and it turns out to be $\simeq 17 \%$. The PF of the cold $B B, \alpha$, is thus completely determined by the measured value of $\mathrm{PF}_{(0.1-1 \mathrm{keV})}$. We obtain $\alpha=10 \pm 1 \%$, a prediction that can be checked once the source returns to the quiescent state.

\subsubsection{The power-law component}

By adopting the $3 B B$ model, we further study the possibility of additional features in the XMM-Newton spectra. In particular, during the first three XMM-Newton observations (2003-2004), the spectral fit residuals suggest the presence of an additional hard component above $7-8 \mathrm{keV}$ ( $3.2 \sigma$ confidence level), which we were not able to characterize because of poor statistics in this 


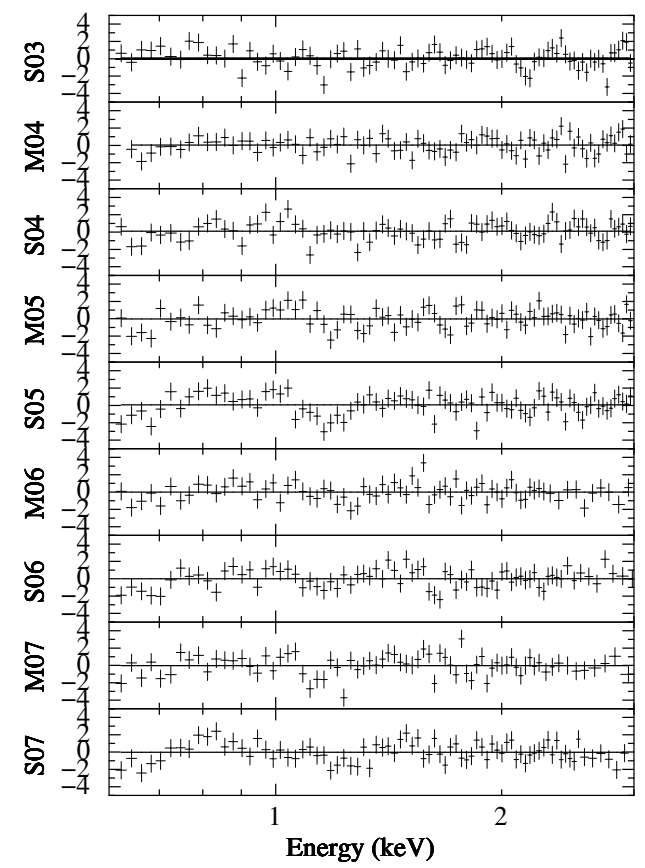

Fig. 4. $\Delta \chi^{2}$ residuals of $3 B B$ model. From March 2005 (M05) onwards is likely present a feature (edge) around $\sim 1.1 \mathrm{keV}$.

band. We can only speculate that it might be related to a hard power-law-like tail $(\Gamma \sim 1.5)$, likely of magnetospheric origin. A similar component has been detected in other AXPs (Kuiper et al. 2004; Kuiper et al. 2006) and extends up to $200 \mathrm{keV}$, at least (Götz et al. 2006). Given the marginal significance of this component, we do not attempt to draw any firm conclusion.

\subsubsection{Narrow spectral feature search}

Starting from the 4th observation (March 2005) we note the presence of excess residuals in the data with respect to the $3 \mathrm{BB}$ model, at around $1.1 \mathrm{keV}$ (Fig. 4). We tried to account for this by including an absorption edge or a Gaussian line in the model. The value of the former is 1 if $E \leq E_{\mathrm{c}}$ and $\exp \left[-\tau_{\max }\left(E / E_{\mathrm{c}}\right)^{-3}\right]$ if $E \geq E_{\mathrm{c}}$, where $E_{\mathrm{c}}$ is the threshold energy and $\tau_{\max }$ the absorption depth at the threshold.

The results of the new spectral model, $3 B B$ plus edge, are consistent with what was obtained with the $3 B B$ model (to within the uncertainties $): N_{\mathrm{H}}=(0.73 \pm 0.02) \times 10^{22} \mathrm{~cm}^{-2}, k T_{\text {cold }}=$ $0.153 \pm 0.005 \mathrm{keV}$, and $R_{\text {cold }}=15.4 \pm 1.8 \mathrm{~km}\left(\chi^{2}=1140\right.$ with d.o.f. $=1038)$. The energy threshold $(\sim 1.1 \mathrm{keV})$ and $\tau_{\max }(\sim 0.2)$ appear to be constant through the latest six observations (Table 1 and Fig. 5).

This new model has $\chi_{\text {red }}^{2}=1.09$, which is $2.2 \sigma_{\chi^{2}}$ from the expectation value. To obtain an estimate of the significance of the edge component we proceeded as follows. We obtained, for each single spectrum, the width of the feature $(\sigma)$ using a Gaussian profile and defined the width at the base of the Gaussian $\left(\Delta E_{\mathrm{i}}\right)$ to be $3 \sigma$. We assumed that the width of the feature is independent of the model used to estimate it. Then we calculated the ratio between the whole spectral range of our data, $\Delta W_{i}$, and the Gaussian width $\Delta E_{\mathrm{i}}$ (number of trials). Finally, to obtain the total probability of the null hypothesis (no line present), we multiplied the probability level $\left(P_{\mathrm{i}}\right)$ attributed by an F-Test to the inclusion of the Gaussian by the number of trials on each spectrum $\left(\Delta W_{\mathrm{i}} / \Delta E_{\mathrm{i}}\right)$ and by the total number of observations (9). The total probability can thus be expressed as $9 \times \Pi_{\mathrm{i}} P_{\mathrm{i}} \Delta W_{\mathrm{i}} / \Delta E_{\mathrm{i}}$,

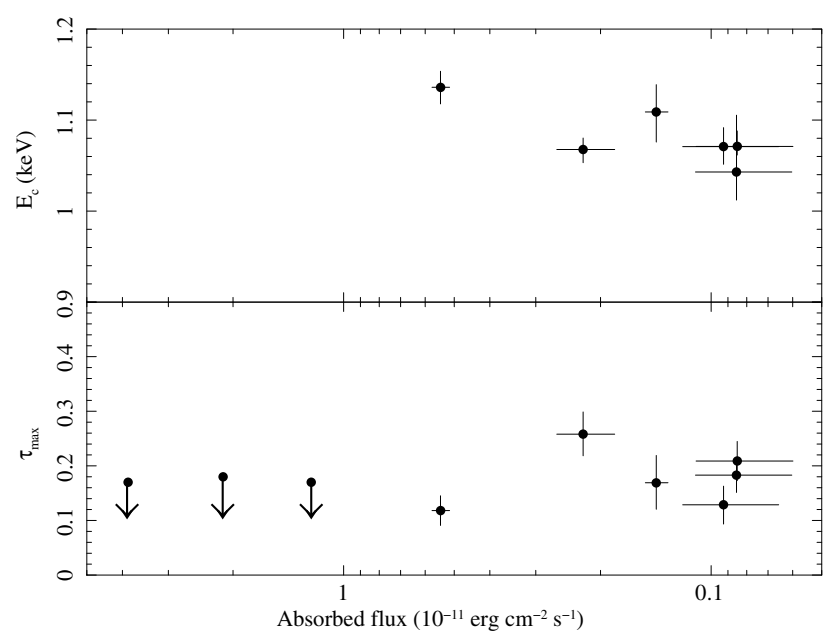

Fig. 5. Parameters of the $\sim 1.1 \mathrm{keV}$ edge (energy $\left(E_{\mathrm{c}}\right)$ and $\tau_{\max }$ ) vs. the observed flux. For the first three observations $\tau_{\max }$ is an upper limit.

which in our case gave a significance for the edge component at the $\simeq 6.5 \sigma$ level.

As a further check, we estimated the line significance by running a Monte Carlo simulation of $10^{5}$ spectra with only the continuum model present (as described in more detail in Rea et al. 2005, 2007). Spectral parameters of the continuum were allowed to vary within 3 sigma from their best fit values and we used the same number of photons of the 4th observation (March 2005). We then counted how many edges, at any energy between $0.5-10 \mathrm{keV}$, with $\tau>0.2$ were significantly detected in the generated spectra just due to statistical fluctuations. We found 12 spectra over $10^{5}$ spectra presenting such a feature, thus leading to an estimated significance level of $\sim 4.1 \sigma$ for our $1.1 \mathrm{keV}$ edge. However, so far we have not considered that the feature has been detected in several spectra rather than only in the 4th observation. To this aim we simulated $10^{5}$ spectra for each of the 6 observations showing the $\sim 1.1$ edge, using the best-fit spectral parameters and the corresponding number of photons for each observation. However, in these simulations we only considered the energy band $0.5-4 \mathrm{keV}$ in which the spectral variability was not very strong among the 6 observations. This reduces to negligible levels any possible systematic error in the probability calculation due to the spectral variability of the source in connection with instrumental response matrices. We estimated the significance of the edge in each observation as described above, then combined them to obtain a total significance of $5.1 \sigma$ for the presence of the line.

By using a Gaussian profile to fit the feature, we obtained results similar to those of the edge component, the mean energy of the feature being $\langle E\rangle \sim 1.15 \mathrm{keV},\langle\sigma\rangle \sim 0.13 \mathrm{keV}$, while the average equivalent width of the line was $\sim 35 \mathrm{eV}\left(\chi_{\text {red }}^{2}=1.09\right.$, 1029 d.o.f).

The use of different chemical abundances for the interstellar medium (ISM), vphabs model in XS PEC, does not produce significant changes in the parameter values or confidence level of the feature, which thus does not seem to depend on the ISM composition.

To test the possible instrumental nature of the feature, we also used the source photons collected by the MOS 1 and MOS2 cameras. As in the case of the PN data, we carried out a spectral analysis by using the $3 B B$ model to account for the continuum spectral component. Table 1 summarizes the results of this test. Starting from the 2005 March observation, the edge 
component is always detected in all three cameras except for the 2005 September observations MOS2 data, where only an upper limit could be obtained. In this case the inferred upper limit is consistent with the values inferred from all other spectra. This finding further supports the interpretation of the edge as intrinsic to the source.

To further check our results, we analyzed the only CHANDRA public observation of XTE J1810-197 made with ACIS, during March 2006 ( $30 \mathrm{ks})$. We used CIAO 4.0 software, a standard reduction procedure, and the last calibration files availeble (3.5.0, 2008 October) for the CHANDRA data analysis. This gives fully consistent results with those obtained with XMM-Newton: $E_{\mathrm{c}}=1.05 \pm 0.3, \tau_{\max }=0.38 \pm 0.9$. The significance for including of this component is $\sim 4.5 \sigma$, determined using the procedure previously exposed. This further confirms the presence of this feature in the continuum of the source. Therefore in the following we consider the $3 B B+$ edge as our best spectral model.

\subsubsection{Other models}

An alternative possibility for modeling the data is to consider the effect of resonant Compton scattering (RCS) in the magnetosphere (Thompson et al. 2002). In this scenario, photons emitted by the star surface, at the temperature of $\sim 0.16 \mathrm{keV}$, are upscattered by energetic electrons and/or positrons in the magnetosphere. Therefore, the increase in X-ray flux during the outburst would not be due (only) to the appearance of (hotter) regions with enhanced emission, but to a shift in energy of upscattered photons.

We performed some tests with a thermal Comptonization model readily available in XS PEC (CompTT, Titarchuk 1994). Although based on completely different physical assumptions from the RCS, this may at least be used to assess whether the observed spectra can be modeled in terms of Comptonization. We fitted together all the 9 spectra, assuming that the plasma temperature is the same at all epochs as a first approximation. A best fit is obtained with an electron temperature of $k T_{\mathrm{e}} \sim 0.8 \mathrm{keV}$, a constant (within uncertainties) temperature for the seed photons of $k T_{\text {seed }} \sim 0.16 \mathrm{keV}$, and a plasma optical depth $\left(\tau_{\mathrm{p}}\right)$ decreasing with time from $\sim 32$ to $\sim 9$. The $\chi_{\text {red }}^{2}$, however, is worse than that of the $3 B B$ model, namely $\chi_{\text {red }}^{2}=1.3$ for 1066 d.o.f. (this value is at $6.7 \sigma_{\chi^{2}}$ from the expectation value). We note also that a scenario in which scattering is (nearly) isotropic and the Comptonizing medium uniformly covers the star surface is hardly compatible with the observed characteristics of the pulsed emission. Indeed, the (relatively) small pulsed fraction of the thermal component would be washed away even more by scattering at higher energies.

Also in this case a feature in the spectrum around $1.1 \mathrm{keV}$ seems to be present. By fitting this feature with an edge component, as in the case of the $3 B B$ model, we obtain a significance level of $\sim 6.5 \sigma$. Therefore, this feature seems to be independent of the model used for the underlying spectral continuum.

\subsection{Pulse-phase spectroscopy}

To understand the role of each spectral parameter in producing the observed $0.6 \div 10 \mathrm{keV}$ flux variation with pulse phase, we carried out a pulse-phase resolved spectroscopic analysis of the XMM-Newton observations with high enough $\mathrm{S} / \mathrm{N}$. The spectra of the first three observations (September 2003-September 2004) were considered and

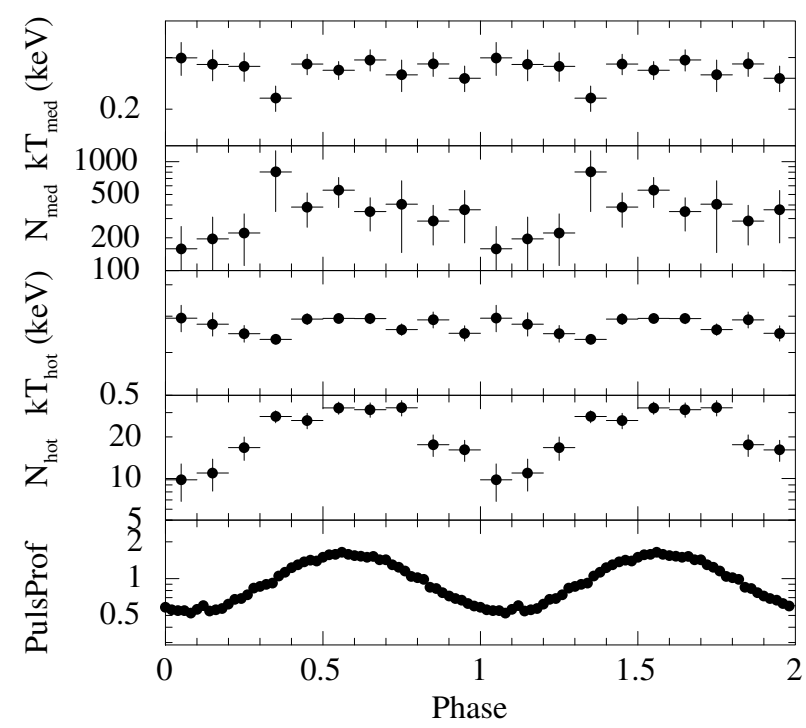

Fig. 6. Phase evolution of $B B_{\text {med }}$ and $B B_{\text {hot }}$ for the 2003 September observation. The two temperatures remained constant to within the uncertainty, while the normalization changed; the ratio $\Delta$ between $N_{\max }$ and $N_{\text {min }}$ is $\Delta N_{\text {hot,med }}=N_{\max } / N_{\min } \sim 3.3$ and both peak at the same pulse phase $(\sim 0.55)$.

divided into 10 phase intervals, in order to rely upon a large enough number of photons. The spectrum in each phase interval was modeled with $3 B B$ s fixing the temperature and radius of $B B_{\text {cold }}$ and $N_{\mathrm{H}}$ at the average values obtained from the previous analysis without including the edge $\left(k T_{\text {cold }}=0.144 \pm 0.003 \mathrm{keV}\right.$; $\left.R_{\text {cold }}=17.9 \pm_{1.5}^{1.9} \mathrm{~km}, N_{\mathrm{H}}=(0.72 \pm 0.02) \times 10^{22} \mathrm{~cm}^{-2}\right)$.

\subsubsection{Pulse-phase spectroscopy with the $3 B B$ model}

In the following we present the results from two representative cases: the 2003 September and 2004 September observations. The PPS analysis of the $B B$ components after the 2004 September pointing was hampered by poor statistics. All parameters were left free to vary except for $k T_{\text {cold }}, R_{\text {cold }}$, and $N_{\mathrm{H}}$, which were frozen at the values reported in Table 4 . We found that the temperature of the medium and hot $B B$ s were nearly constant through the whole pulse cycle, whereas the normalization/emitting-area were clearly variable (see Fig. 6). To study these variations better, we fixed the $B B$ temperatures at their phase-averaged values, leaving only the normalizations $(N)$ of the spectra free to vary ${ }^{3}$. These were then converted into the radii of the $B B$ components $\left(R_{\mathrm{BB}}=\sqrt{N} \times D_{10}\right.$, assuming a source distance of $3.5 \mathrm{kpc}$ ). Figures 7, 8, and Table 5 show our results. In particular, in 2003 September the ratio $\Delta=R_{\max } / R_{\min }$ for each component was $\Delta R_{\text {hot }}=1.8 \pm 0.1$ and $\Delta R_{\text {med }}=1.5 \pm 0.2$, and $\Delta R_{\text {hot }}=1.3 \pm 0.1, \Delta R_{\text {med }}=1.2 \pm 0.1$ in 2004 September. In both cases, the modulation of the radii $(R)$ with phase only shows one peak for each pulse cycle. Moreover, they appear to be phase-aligned with each other and with the peak of the total pulse profile. This suggests that the two $B B$ regions must be relatively close to each other and probably connected, otherwise a phase lag/shift would naturally be expected. The $R$-variation amplitude as a function of phase is more pronounced at higher energies, in agreement with the timing properties of this pulsar, where the pulsed fraction is greater at higher energies.

\footnotetext{
3 In the "blackbodyrad" model the normalization is $N=R_{\mathrm{km}}^{2} / D_{10}^{2}$, with $D_{10}=0.35$ the distance to the source in units of $10 \mathrm{kpc}$.
} 
Table 4. Temperature, radius and observed flux $(0.6-10 \mathrm{keV})$ evolution with time of $B B$ medium (med) and hot $B B$ (hot) in the $3 B B$ model with $1 \sigma$ uncertainties.

\begin{tabular}{cccc}
\hline \hline Epoch & $\begin{array}{c}k T_{\text {med }} \\
\mathrm{keV}\end{array}$ & $\begin{array}{c}R_{\text {med }} \\
\mathrm{km}\end{array}$ & $\begin{array}{c}F_{\text {med }} \\
\mathrm{erg} \mathrm{s}^{-1} \mathrm{~cm}^{-2}\end{array}$ \\
\hline Sep. 03 & $0.267 \pm 0.009$ & $6.9 \pm 0.6$ & $(5 \pm 1) \times 10^{-12}$ \\
Mar. 04 & $0.29 \pm 0.01$ & $4.9 \pm 0.4$ & $(4 \pm 1) \times 10^{-12}$ \\
Sep. 04 & $0.271 \pm 0.006$ & $4.8 \pm 0.3$ & $(2.6 \pm 0.5) \times 10^{-12}$ \\
Mar. 05 & $0.264 \pm 0.007$ & $3.9 \pm 0.3$ & $(1.5 \pm 0.3) \times 10^{-12}$ \\
Sep. 05 & $0.280 \pm 0.009$ & $2.6 \pm 0.2$ & $(9 \pm 2) \times 10^{-13}$ \\
Mar. 06 & $0.28 \pm 0.01$ & $2.0 \pm 0.2$ & $(6 \pm 2) \times 10^{-13}$ \\
Sep. 06 & $0.304 \pm 0.006$ & $1.5 \pm 0.1$ & $(5 \pm 1) \times 10^{-13}$ \\
Mar. 07 & $0.296 \pm 0.006$ & $1.5 \pm 0.2$ & $(4 \pm 1) \times 10^{-13}$ \\
Sep. 07 & $0.308 \pm 0.006$ & $1.3 \pm 0.1$ & $(4 \pm 1) \times 10^{-13}$ \\
Sep. 06-07 & $0.301 \pm 0.003$ & $1.42 \pm 0.03$ & $(4.1 \pm 0.2) \times 10^{-13}$ \\
& & & \\
Epoch & $k T_{\text {hot }}$ & $R_{\text {hot }}$ & $\begin{array}{c}F_{\text {hot }} \\
\text { km }\end{array}$ \\
Sep. 03 & $0.681 \pm 0.005$ & $1.58 \pm 0.04$ & $(3.3 \pm 0.2) \times 10^{-11}$ \\
Mar. 04 & $0.70 \pm 0.01$ & $1.07 \pm 0.04$ & $(1.7 \pm 0.2) \times 10^{-11}$ \\
Sep. 04 & $0.68 \pm 0.05$ & $0.83 \pm 0.02$ & $(9.2 \pm 0.7) \times 10^{-12}$ \\
Mar. 05 & $0.61 \pm 0.08$ & $0.66 \pm 0.03$ & $(3.5 \pm 0.5) \times 10^{-12}$ \\
Sep. 05 & $0.62 \pm 0.03$ & $0.31 \pm 0.05$ & $(8 \pm 4) \times 10^{-13}$ \\
Mar. 06 & $0.61 \pm 0.05$ & $0.22 \pm 0.07$ & $(4 \pm 3) \times 10^{-13}$ \\
Sep. 06 & $0.65^{a}$ & $<0.08$ & $<8.7 \times 10^{-14}$ \\
Mar. 07 & $0.65^{a}$ & $<0.06$ & $<6.1 \times 10^{-14}$ \\
Sep. 07 & $0.65^{a}$ & $<0.06$ & $<5.4 \times 10^{-14}$ \\
Sep. 06-07 & $0.655^{a}$ & $<0.05$ & $<4.5 \times 10^{-14}$ \\
\hline & & &
\end{tabular}

${ }^{a}$ Fixed to the average of the earliest measurements. Upper limits are inferred at $3 \sigma$ confidence level. $k T_{\text {cold }}=0.144 \pm 0.003 \mathrm{keV} ; R_{\text {cold }}=$ $17.9 \pm_{1.5}^{1.9} \mathrm{~km}, N_{\mathrm{H}}=(0.72 \pm 0.02) \times 10^{22} \mathrm{~cm}^{-2}$ are constant throughout the outburst.

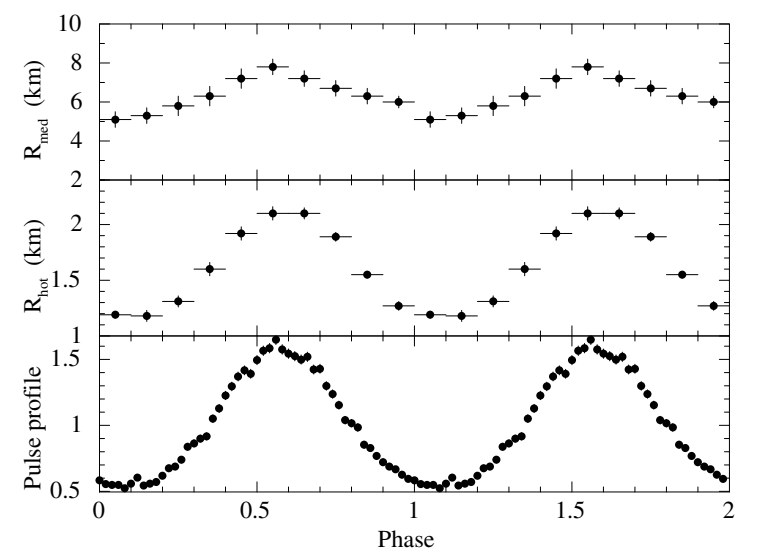

Fig. 7. $R_{\text {med }}$ and $R_{\text {hot }}$ as a function of rotation phase for the 2003 September observation. The temperatures $k T_{\text {med }}$ and $k T_{\text {hot }}$ are held fixed at the phase average value listed in Table 4 , while the normalization constant (which is related to the radius) is left free to vary.

\subsubsection{Pulse phase spectroscopy of the $\sim 1.1 \mathrm{keV}$ edge}

A similar analysis was carried out for the narrow spectral feature detected in the spectra from the 2005 March observation onwards. Given the relatively small number of photons, we reduced the number of phase intervals to five and kept the spectral parameters of the coldest $B B$ fixed ( $3 B B+$ edge model value). In Fig. 9 and Table 6 we report the result for the 2005 September observation, when there was a possible indication that the component evolved with phase. Although the value of $\tau_{\max }$ is compatible with being constant $\left(\chi^{2}=6.57\right.$ with 4 d.o.f.), we note that it varies from a minimum of $0.13 \pm 0.06$ to a maximum

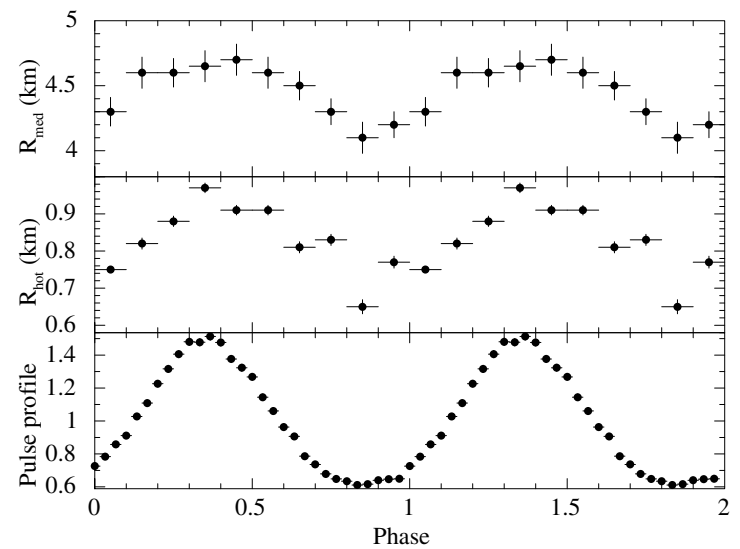

Fig. 8. $R_{\text {med }}$ and $R_{\text {hot }}$ as a function of rotation phase for the 2004 September observation. The temperatures $k T_{\text {med }}$ and $k T_{\text {hot }}$ are held fixed at the phase-averaged value listed in Table 4, while the normalization constant (which is related to the radius) is set free to vary.

Table 5. $R_{\text {med }}$ and $R_{\text {hot }}$ as a function of pulse phase for the 2003 September and 2004 September observations.

\begin{tabular}{ccccc}
\hline \hline Phase bin & $\begin{array}{c}\text { Sep. 03 } \\
R_{\text {med }}{ }^{a} \\
\mathrm{~km}\end{array}$ & $\begin{array}{c}\text { Sep. 03 } \\
R_{\text {hot }} \\
\mathrm{km}\end{array}$ & $\begin{array}{c}\text { Sep. 04 } \\
R_{\text {med }} \\
\mathrm{km}\end{array}$ & $\begin{array}{c}\text { Sep. 04 } \\
R_{\text {hot }} \\
\mathrm{km}\end{array}$ \\
\hline $0.0-0.1$ & $5.1 \pm 0.4$ & $1.19 \pm 0.03$ & $4.3 \pm 0.1$ & $0.750 \pm 0.001$ \\
$0.1-0.2$ & $5.3 \pm 0.4$ & $1.18 \pm 0.05$ & $4.6 \pm 0.1$ & $0.82 \pm 0.02$ \\
$0.2-0.3$ & $5.8 \pm 0.5$ & $1.31 \pm 0.05$ & $4.6 \pm 0.1$ & $0.88 \pm 0.01$ \\
$0.3-0.4$ & $6.3 \pm 0.5$ & $1.60 \pm 0.06$ & $4.6 \pm 0.1$ & $0.97 \pm 0.01$ \\
$0.4-0.5$ & $7.2 \pm 0.5$ & $1.92 \pm 0.06$ & $4.7 \pm 0.1$ & $0.91 \pm 0.01$ \\
$0.5-0.6$ & $7.8 \pm 0.4$ & $2.10 \pm 0.06$ & $4.6 \pm 0.1$ & $0.91 \pm 0.01$ \\
$0.6-0.7$ & $7.2 \pm 0.4$ & $2.10 \pm 0.05$ & $4.5 \pm 0.1$ & $0.81 \pm 0.02$ \\
$0.7-0.8$ & $6.7 \pm 0.4$ & $1.89 \pm 0.04$ & $4.3 \pm 0.1$ & $0.83 \pm 0.02$ \\
$0.8-0.9$ & $6.3 \pm 0.4$ & $1.55 \pm 0.03$ & $4.1 \pm 0.1$ & $0.65 \pm 0.02$ \\
$0.9-0.1$ & $6.0 \pm 0.3$ & $1.27 \pm 0.04$ & $4.2 \pm 0.1$ & $0.77 \pm 0.02$ \\
\hline
\end{tabular}

${ }^{a}$ Temperatures are kept fixed at the value listed in Table 4. $1 \sigma$ confidence level uncertainties are given. The corresponding $\chi_{\text {red }}^{2}$ are 0.92 (for 852 d.o.f.) and 1.01 (1132 d.o.f.) for September 2003 and 2004, respectively.

of $0.31 \pm 0.07$ in a smooth way, which we tried to model with a simple sinusoidal function. An F-test for the addition of the sinusoid gave just a marginal detection $(\sim 2.4 \sigma)$, hence no claim can be made about its actual presence; however this possible modulation is worth further investigation with deeper observations.

\section{Discussion}

The spectral and temporal information obtained from the nine XMM-Newton observations of the transient anomalous X-ray pulsar XTEJ1810-197 collected in 2003-2007 allowed us to study the source behavior during the outburst to an unprecedented level of detail. As discussed below, our results shed some light on several issues concerning the mechanism powering the emission during the active period. During four years of monitoring, the X-ray flux of XTE J1810-197 continued to decrease following an almost exponential decay. In September 2007 the source nearly reached its quiescent emission level as recorded by ROSAT in 1992. In the following we summarize the most relevant findings obtained from the XMM-Newton dataset. 


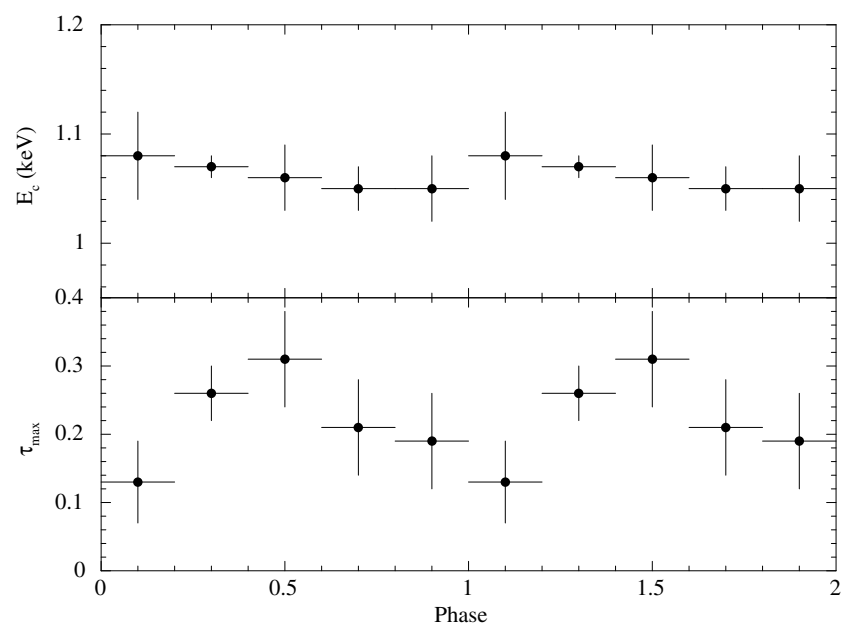

Fig. 9. Evolution of the edge parameters $E_{\mathrm{c}}$, and $\tau_{\max }$ with pulse phase for the 2005 September observation ( $1 \sigma$ confidence level uncertainties are reported).

Table 6. Evolution of the spectral parameters for the edge component as a function of phase for the 2005 September observation (PN data) with uncertainties at $1 \sigma$ confidence level.

\begin{tabular}{ccc}
\hline \hline Phase bin $^{a}$ & $\begin{array}{c}E \\
\mathrm{keV}\end{array}$ & $\tau_{\max }$ \\
\hline $0.0-0.2$ & $1.08 \pm 0.04$ & $0.13 \pm 0.06$ \\
$0.2-0.4$ & $1.07 \pm 0.01$ & $0.26 \pm 0.04$ \\
$0.4-0.6$ & $1.06 \pm 0.03$ & $0.31 \pm 0.07$ \\
$0.6-0.8$ & $1.05 \pm 0.02$ & $0.21 \pm 0.07$ \\
$0.8-1.0$ & $1.05 \pm 0.03$ & $0.13 \pm 0.07$ \\
\hline
\end{tabular}

${ }^{a}$ The five spectra are fitted together and result in a $\chi_{\text {red }}^{2}$ of 1.01 (for 406 d.o.f.)

\subsection{The continuum spectral component}

We find that the previously proposed $2 B B$ model for the source spectrum during the outburst fails to account for the time evolution of the hot-temperature spectral components and for the PF flattening (see Sect. 3.2.1). Similar concerns have already been expressed first by Israel et al. (2007b) and after by Perna \& Gotthelf (2008), but see e.g. Güver et al. (2007) for a different interpretation. For these reasons we included a third softer thermal component that, as we have shown in the previous sections, improves the spectral fits and also removes the inconsistency that appears in the $2 B B$ model when the evolution of the PF is considered. The temperature and radius of this additional $B B$ turn out to be the same as those inferred from the XTE J1810-197 ROSAT spectra serendipitously collected since 1992, when the source was in quiescence.

The additional $B B$ component is compatible with being emitted from the whole NS surface and appears to be unaffected by the outburst. Therefore, its nearly constant flux can be taken as representative the minimum level of emission from the source. It also provides the key to understanding the previously unexplained PF flattening. We emphasize that the $3 B B$ model discussed here should be regarded as a crude, albeit convenient, description of a scenario in which other effects may come into play (see below). Nevertheless, it has the advantage of being independent of the often poorly known details of the atmosphere/magnetosphere of the neutron star. As such, it provides a first estimate of some key physical parameters, like the size and temperatures of the emitting region(s), without relying on any assumptions about the field strength or geometry. Intriguingly, our analysis reveals that only the size of the hot/warm regions varied during the outburst, showing an almost steady decrease, while the temperatures remained nearly constant.

Although present data do not allow us to tightly constrain the shape and relative position of the hot and medium temperature regions on the star, a simple model can be used to gain some insight into the geometry of the source. We assume that emission comes from two concentric zones: an inner, hot cap, and an outer, warm corona, outside of which is the colder surface of the star at $T_{\text {cold }}=0.160 \mathrm{keV}^{4}$, a picture very similar to that adopted by Israel et al. (2007b). For a NS of $1.4 M_{\odot}$ and typical NS radii, we computed the PFs after applying the proper relativistic corrections. Since the angular (semi)aperture of the two zones follows from the values of the blackbody radii, and their temperatures are just $k T_{\text {hot }}$ and $k T_{\text {med }}$, the only free parameter is the angle between the diameter through the cap center and the rotation axis, i.e. the cap's colatitude. The observed spectrum and the lightcurves also depend on the angle between the line of sight (LOS) and the rotation axis. Without performing any formal fit, we simply tried various combinations of these angles, and we find that there is reasonable agreement between PF data and the model at all epochs for values that are consistent with the range determined by the detailed analysis of Perna \& Gotthelf (2008; see also Kramer et al. 2007, for constraints on the pulsar geometry through radio polarimetry). This is expected since the spectrum in the first 4 epochs, which Perna \& Gotthelf analyzed, is not very affected by the emission from the coldest part of the star surface.

We note that our analysis based on the $3 B B$ model suggests that the coldest $B B$ component, accounting for the emission from the whole surface, has a low pulsed fraction, $10 \% \pm 1 \%$. If our model is correct, this prediction can be checked once the source returns to the quiescent state. We also note that this value is similar to that found in X-ray Dim Isolated Neutron Stars (XDINSs), where it is believed that the (purely) thermal emission comes from the cooling NS surface (e.g. Haberl 2007). Although magnetars as a class are probably far from being passive coolers, this similarity makes a case for our interpretation of the cold $B B$ component as the quiescent emission from the NS surface, worth being pursued in future studies.

On the other hand, the narrower pulse profile and higher pulsed fraction at increasing energies seems reminiscent of what has been found for other AXPs with RXTE and INTEGRAL in the energy band above $10 \mathrm{keV}$. Indeed, the narrowing of the peak is coincident with the emergence of a hard power-law component extending from $10-20 \mathrm{keV}$ up to $200 \mathrm{keV}$ at least (Kuiper et al. 2004). The origin of this component is most likely magnetospheric. The marginal detection, during the first three $X M M$-Newton pointings, of a possible hard power-law tail extending above $10 \mathrm{keV}$, corroborates this reasoning. However we could not study the power-law tail in more detail, due to insufficient statistics.

We performed also a preliminary test with a different model, a simple Comptonization model available in XS PEC (CompTT), but this gives a worse fit for the data than does the $3 B B$ model. More advanced RCS models in which the optical depth is provided by currents flowing in a twisted magnetosphere (Lyutikov \& Gavriil 2006; Fernandez \& Thompson 2007; Nobili et al. 2008a,b) appear, on the other hand, promising in explaining the pulse profiles, since the particle density changes with the magnetic colatitude, increasing as one moves from the

${ }_{4}$ The radii of these regions are taken from Table 3. 
magnetic pole towards the equator. Such a distribution naturally introduces a pulsed fraction even when the surface temperature is homogeneous, as recently shown on the basis of Montecarlo simulations by Nobili et al. (2008a) and Pavan et al. (in preparation). A first attempt to systematically apply RCS to all AXPs, including XTE J1810-197 is reported by Rea et al. (2008). These authors find that the outburst of this source may result from heating of the NS surface, which slowly cools on a timescale of months/years, while the magnetospheric properties only show a small variation during the outburst decay.

\subsection{The narrow feature at $\sim 1 \mathrm{keV}$}

Within the framework of the magnetar model, a natural interpretation for the absorption-like feature that is significantly detected in the PN and MOS spectra is that it stems from to a proton cyclotron line. The observation of such a feature would directly probe the magnetic field strength of the AXP, since the line energy is proportional to the field strength:

$E_{\text {cyc }}=0.63(1+z)^{-1}\left(\frac{B}{10^{14} G}\right) \mathrm{keV}$

where $(1+z)^{-1}=\left(1-2 G M / R c^{2}\right)^{\frac{1}{2}} \simeq 0.8$ is the gravitational redshift at the neutron star surface. Here we assume $M=1.4 M_{\odot}$ and $R=10 \mathrm{~km}$ for the star mass and radius. Despite a few earlier claims (Ibrahim et al. 2002; Rea et al. 2003), unambiguous evidence of absorption lines in the spectra of magnetars has not yet been obtained.

If the edge detected in the XTE J1810-197 spectra is a proton cyclotron feature, its energy implies $2.1 \times 10^{14} \mathrm{G} \leq B_{\text {prot }} \leq 2.6 \times$ $10^{14} \mathrm{G}$ when taken at face value. On the other hand, the assumption of a constant field breaks down if the line originates in a relatively large region on the neutron star surface/magnetosphere. For instance, Zane et al. (2001) estimate that, even for a simple dipolar field, that $B$ changes in both magnitude and direction will produce a broadening of a feature that is emitted by the whole surface (typically by $10 \%-20 \%$ ) and a shift in the line centroid toward lower energies by $20 \%-30 \%$ with respect to the prediction based on Eq. (1).

Similar absorption features are also observed in the spectra of XDINSs (Haberl 2007) and are typically associated with proton cyclotron and/or bound-free, bound-bound transitions in $\mathrm{H}$, $\mathrm{H}$-like and He-like atoms in the presence of relatively high magnetic fields $B \approx 5 \times 10^{13}-10^{14} \mathrm{G}$ (e.g. van Kerkwijk \& Kaplan 2007; Ho et al. 2003; Pavlov \& Bezchastnov 2005). At such large field strengths, exotic molecules might also contribute to line formation (Turbiner et al. 2007; Turbiner \& Lopez-Vieyra 2006). For XDINSs, all the above mentioned scenarios provide similar values of $B$, which turns out to be in agreement with those derived from the spin-down rate (e.g. Kaplan 2008). A similar absorption feature has been discovered in the spectrum of the Rotating RAdio Transient (RRAT) detected at X-ray energies, J1819-1458 (McLaughlin et al. 2007). The X-ray spectrum of RRAT J1819-1458 is well-fit by an absorbed blackbody with $k T=0.14 \mathrm{keV}$ with the addition of an absorption feature at $\sim 1 \mathrm{keV}$, which, when interpreted either as a proton cyclotron line or as an atomic transition, yields a magnetic field of $5 \times 10^{13} \mathrm{G}$, again in rough agreement with the spin-down measure (McLaughlin et al. 2007). In the case of XTE J1810-197, the magnetic field value inferred by using Eq. (1) also appears to be in very good agreement with that obtained through the spindown measurement: $2.2 \times 10^{14} \mathrm{G} \leq B_{\text {dip }} \leq 3.1 \times 10^{14} \mathrm{G}$. It is interesting to note that a similar value, $B=(2.72 \pm 0.03) \times 10^{14} \mathrm{G}$, was

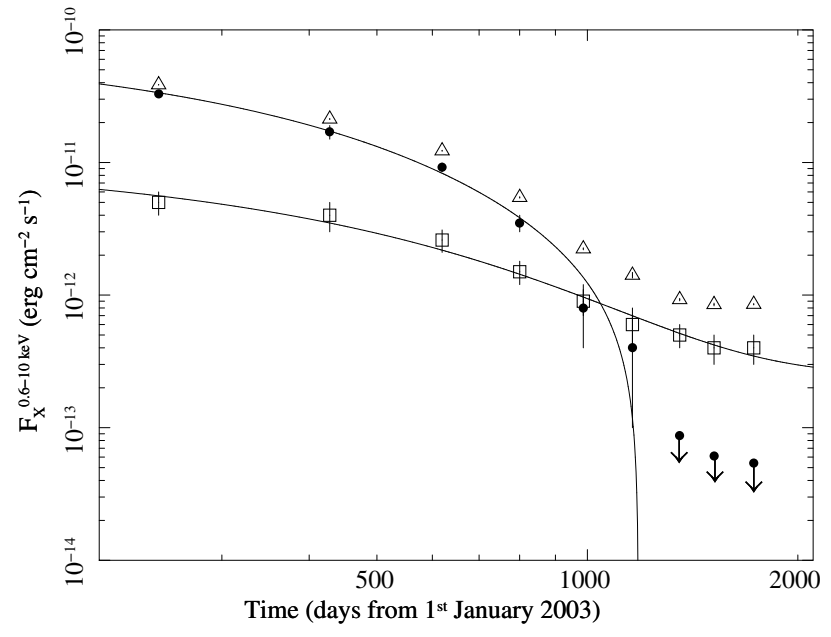

Fig. 10. Evolution of the $0.6-10 \mathrm{keV}$ flux (as measured with the EPIC/PN camera onboard XMM-Newton of XTE J1810-197 as a function of time, for the $B B_{\text {med }}$ (squares), the $B B_{\text {hot }}$ (circles), and the sum of the $3 B B$ s (triangles). The solid line represents the best fit obtained for the $B B_{\text {med }}$ and $3 B B$ evolution by using a model consisting of an exponential decay plus a constant.

obtained by Güver et al. (2007) based on the 2003 September2006 March XMM-Newton spectrum of XTE J1810-197. It is worth emphasizing that the spectral model used by Güver et al. (2007), has been specifically developed for passively cooling NS and magnetic field stronger than $5 \times 10^{13} \mathrm{G}$ and is, therefore, rather different from the $3 B B$ model adopted here.

A different possibility is that the line stems from the presence of Iron in proximity to the star surface. In particular, L shell electronic transitions of Iron ions XXII, XXIII, XXIV, have energies between 1.05 and $1.17 \mathrm{keV}$. However, this requires that the line absorbing region is permeated by a relatively low magnetic field. Future, longer observations, with much higher statistics, might help to understand the nature of this spectral feature better.

\subsection{Flux evolution}

During approximately four years of XMM-Newton monitoring, the X-ray flux of XTE J1810-197 continued to decrease and is presently $\sim 15 \%-20 \%$ above the quiescent level (as determined by $R O S A T$ ). In Fig. 10 the evolution of the total X-ray flux in the $0.6-10 \mathrm{keV}$ band is shown, together with the flux evolution of the two hotter $B B \mathrm{~s}, B B_{\text {med }}$ and $B B_{\text {hot }}$. Notably, both the $B B_{\text {med }}$ and $B B_{\text {hot }}$ flux evolutions are well-fit by an exponential decay plus a constant $\left(\chi^{2}=2\right.$ for 5 d.o.f. and $\chi^{2}=5$ for 2 d.o.f., respectively). The characteristic times are $\tau=370 \pm 40$ days and $\tau=250 \pm 10$ days for $B B_{\text {med }}$ and $B B_{\text {hot }}$, respectively. This might hint at a common physical process responsible for the decay of the two $B B$ components, though on slightly different timescales. A possible flattening in the $B B_{\text {med }}$ flux evolution, as suggested by the latest two/three flux measurements, might imply that this component has already reached its quiescent state (see discussion below).

To test this hypothesis further, we superimposed the average spectral model, referring to the latest three XMM-Newton observations (September 2006-2007, where only the $B B_{\text {cold }}$ and $B B_{\text {med }}$ components are detected), to the average ROSAT spectrum obtained by merging the three longest pointings (total effective exposure of $\sim 22 \mathrm{ks}$ ). This model is compared with the single $B B$ model used so far for the ROSAT data. The result of 


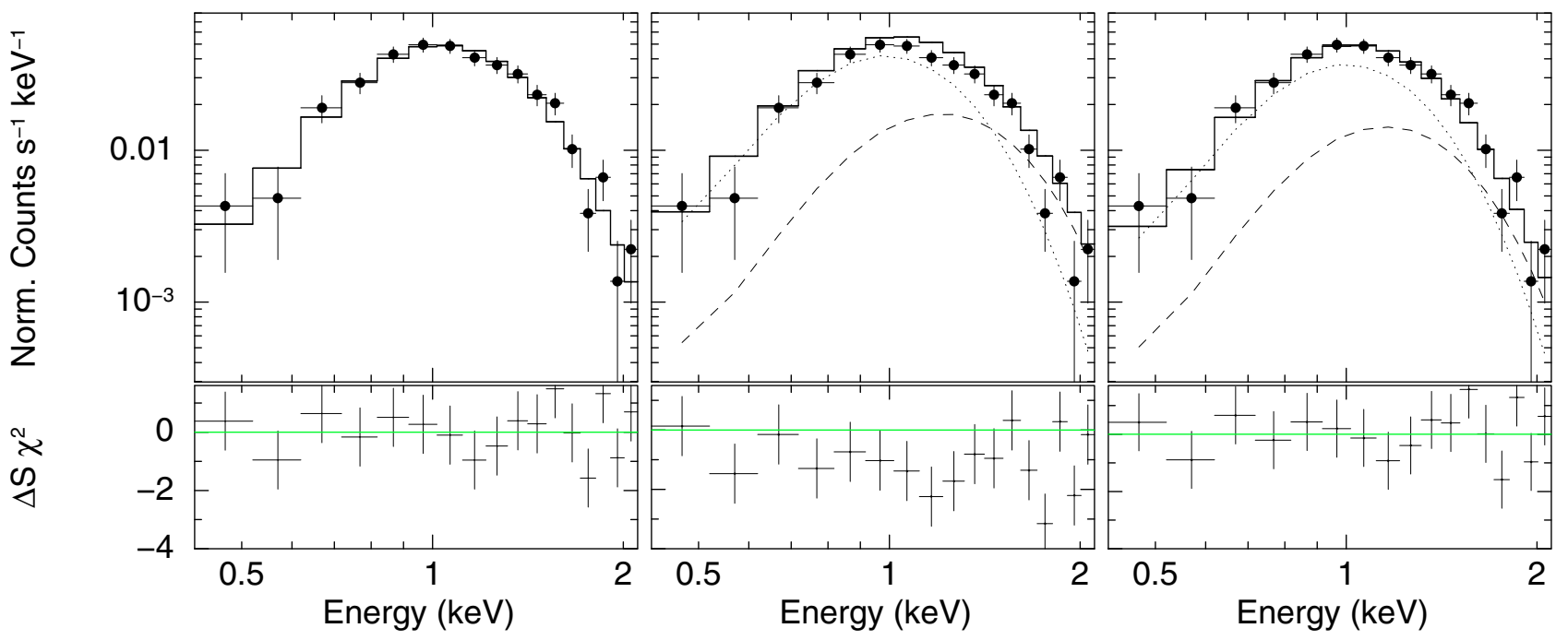

Fig. 11. ROSAT PSPC spectrum of the pre-outburst quiescent state of XTE J1810-197 (three PSPC observations were merged to rely upon a higher $\mathrm{S} / \mathrm{N}$ ), fitted with one $B B$ component (left panel), the same ROSAT spectrum with superimposed the $2 B B$ model inferred from the latest three $X M M$-Newton observations (since September 2006 the $B B_{\text {hot }}$ is not detected; no fit was performed; middle panel), same as before, but leaving the $2 B B$ model parameters free to vary (right panel). The dotted and dash-stepped lines mark the $B B_{\text {cold }}$ and $B B_{\text {med }}$ components, respectively.

this test is shown in Fig. 11. It is evident from the first and second panels that the September 2006-2007 XMM-Newton model agrees with the ROSAT data in considering that no fit has been performed, suggesting that the source might already be back to its quiescent state after March 2007. If correct, the quiescent state of XTE J1810-197 could be characterized by two BBs instead of one $B B$ as discussed so far. However, we emphasize that including of the second $B B$ in the ROSAT spectral fit is formally not statistically required. In fact, we reanalyzed the ROSAT data by using either a single $B B$ or a $2 B B$ model, and in both cases obtained a $\chi_{\text {red }}^{2}=0.9$ (left and right panels of Fig. 11). Thebest fit parameters are: $(B B) N_{\mathrm{H}}=(0.63 \pm 0.05) \times 10^{22} \mathrm{~cm}^{-2}$, $k T=0.19 \pm 0.03 \mathrm{keV}$, and $R<11 \mathrm{~km}\left(\chi^{2}=13\right.$ for 14 d.o.f. $)$, $(2 B B) N_{\mathrm{H}}=(0.75 \pm 0.08) \times 10^{22} \mathrm{~cm}^{-2}, k T_{\text {cold }}=0.16 \pm 0.03 \mathrm{keV}$ and $R_{\text {cold }}=16 \pm 5 \mathrm{~km}, k T_{\text {med }}=0.26 \pm 0.06 \mathrm{keV}$ and $R_{\text {med }}<5 \mathrm{~km}$ $\left(\chi^{2}=11\right.$ for 12 d.o.f.). On the other hand, we note that the $X M M-N e w t o n$ model remains slightly above the ROSAT data mainly around $1 \mathrm{keV}$, where the $B B_{\text {med }}$ component is maximum. This might suggest that the flux of the latter component is still decaying. Clearly, deeper and higher statistics observation of XTE J1810-197 at some later time might solve this issue.

\section{Conclusions}

In this paper we reported the detailed timing and spectral analysis of a long-term (4 years) XMM-Newton monitoring program aimed at unveiling the physical processes responsible for the decaying phases of the XTE J1810-197 outburst. The main results can be summarized as follows:

- We find that a spectral model with three blackbodies much better agrees with the data than the previously used model involving two blackbodies. Also, the $3 \mathrm{BB}$ model solves several ambiguities in the spectral evolution that were present in the $2 B B$ model.

- The best spectral fit at the different epochs is obtained for three blackbodies plus an edge. The best-fit spectral parameters determined with this model are: $k T_{\text {cold }} \sim 0.15 \mathrm{keV}$ and $R_{\text {cold }} \sim 15 \mathrm{~km}$. The latter feature is required starting from the 2005 March observation, where residuals with respect to the simple $3 B B$ model are clearly recognized. The coldest $B B$ component temperature and emitting radius remain constant during the whole outburst and are the same as those of the single $B B$ component observed by ROSAT, which is very likely emitted from the whole NS surface. The two hotter and smaller regions ( $\sim 5$ and $\sim 1 \mathrm{~km})$ evolve in size but, again, at constant temperature. The emitting surface decreases in both cases and these components are, therefore, probably responsible for the enhancement of the observed X-ray flux during the outburst.

Since September 2006 the hottest component, $B B_{\text {hot }}$, is no longer needed in the fit and the $3 B B$ model evolves into a $2 B B$ model. At the same epoch, the average pulsed fraction of the $5.54 \mathrm{~s}$ modulation levels up suggesting that the greatest part of the pulsed photons were produced in the $B B_{\text {hot }}$ component.

- During the first three XMM-Newton observations (20032004), the spectral fit residuals suggest the presence of an additional component above $7-8 \mathrm{keV}$, probably a hard tail, possibly similar to the one detected in other AXPs (where it extends up to $200 \mathrm{keV}$ ). The limited sensitivity of the EPIC cameras above $10 \mathrm{keV}$ prevented us from performing a detailed analysis of this component.

- By assuming that the feature around $1.1 \mathrm{keV}$ is due to a proton cyclotron resonance, we obtain a surface magnetic field value of $2.1 \times 10^{14} \mathrm{G} \leq B_{\text {prot }} \leq 2.6 \times 10^{14} \mathrm{G}$. This estimate is in very good agreement with that obtained from the spindown measure of $1.6 \times 10^{14} \mathrm{G} \leq B_{\text {dip }} \leq 2.8 \times 10^{14} \mathrm{G}$. We can't currently exclude that the absorption feature originates from $L$-shell transitions of Fe XXII, XXIII, and XXIV.

- The analysis of the pulsed fraction time evolution as a function of energy shows an increase with energy, within individual observations, and a decrease as a function of time, within the same energy interval. Most of the modulation is ascribed to high-energy photons coming from the two hottest $B B$ emitting regions.

- Pulse-phase spectroscopy shows that emission from the two hotter $B B$ s peaks at the same phase interval, suggesting that they are emitted by close regions (e.g. two concentric zones). 
- The observed (0.6-10 keV) flux evolution of the $B B_{\text {med }}$ and $B B_{\text {hot }}$ is described well by an exponential decay, with characteristic times of $\tau=370 \pm 40$ days and $\tau=250 \pm 10$ days, respectively. This suggests that the same physical process is responsible for the decay of the two thermal components, as already noted by Gotthelf \& Halpern (2005). While, in a $2 B B$ model, the hot component shows similar time decay ( $\tau \sim 300$ days), the decay time of the colder one is longer ( $\sim 900$ days). This agrees with the presence of a colder component, emitted by the whole star surface.

- A comparison between the latest three XMM-Newton pointings and a re-analysis of the ROSAT quiescent spectrum reveals that the $B B_{\text {med }}$ component might have already reached its quiescent state.

Acknowledgements. This work is partially supported at OAR through Agenzia Spaziale Italiana (ASI), Ministero dell'Istruzione, Università e Ricerca Scientifica e Tecnologica (MIUR - COFIN), and Istituto Nazionale di Astrofisica (INAF) grants. We acknowledge financial contribution from contract ASIINAF I/023/05/0 and AAE TH-058. Based on observations obtained with $X M M-N e w t o n$, an ESA science mission with instruments and contributions directly funded by ESA Member States and NASA. This research has made use of data obtained through the High Energy Astrophysics Science Archive Research Center Online Service, provided by the NASA/Goddard Space Flight Center. S.Z. acknowledges support from a STFC (ex-PPARC) A.F. D.G. acknowledges financial support from the French Space Agency (CNES).

\section{References}

Camilo, F., Ransom, S. M., Halpern, J. P., et al. 2006, Nature, 442, 892 Camilo, F., Ransom, S. M., Peñalver, J., et al. 2007a, ApJ, 669, 561 Camilo, F., Ransom, S. M., Halpern, J. P., \& Reynolds, J. 2007b, ApJ, 666, L93 Camilo, F., Cognard, I., Ransom, S. M., et al. 2007c, ApJ, 663, 497 den Herder, J. W., Brinkman, A. C., Kahn, S. M., et al. 2001, A\&A, 365, L7 Brinkman, A. C., Kahn, S., MDall'Osso, S., et al. 2003, ApJ, 599, 485 Duncan, R. C., \& Thompson, C. 1992, ApJ, 392, L9 Fernández, R., \& Thompson, C. 2007, ApJ, 660, 615 Gavriil, F. P., Kaspi, V. M., \& Woods, P. M. 2004, ApJ, 607, 959 Gelfand, J. D., \& Gaensler, B. M. 2007, ApJ, 667, 1111 Gotthelf, E. V., \& Halpern, J. P. 2005, ApJ, 632, 1075

Gotthelf, E. V., \& Halpern, J. P. 2007, Ap\&SS, 308, 79 Gotthelf, E. V., Halpern, J. P., Buxton, M., \& Bailyn, C. 2004, ApJ, 605, 368 Götz, D., Mereghetti, S., Tiengo, A., \& Esposito, P. 2006, A\&A, 449, L31 Güver, T., Özel, F., Gögüuss, E., \& Kouveliotou, C. 2007, ApJ, 667, L73 Haberl, F. 2007, ApS\&S, 308, 181

Halpern, J. P., Gotthelf, E. V., Becker, R. H., Helfand, D. J., \& White, R. L. 2005, ApJ, 632, L29
Halpern, J. P., Gotthelf, E. V., Reynolds, J., Ransom, S. M., \& Camilo, F. 2008, ApJ, 676, 1178

Helfand, D. J., Chatterjee, S., Brisken, W. F., et al. 2007, ApJ, 662, 1198

Ho, W. C. G., Lai, D., Potekhin, A. Y., \& Chabrier, G. 2003, ApJ, 599, 1293

Ibrahim, A. I., Safi-Harb, S., Swank, J., et al. 2002, ApJ, 574, L51

Ibrahim, A. I., Markwardt, C. B., Swank, J. H., et al. 2004, ApJ, 609, L21

Israel, G. L., Rea, N., Mangano, V., et al. 2004, ApJ, 603, L97

Israel, G., Covino, S., Mignani, R., et al. 2005, A\&A, 438, L1

Israel, G. L., Campana, S., Dall'Osso, S., et al. 2007a, ApJ, 664, 448

Israel, G. L., Bernardini, F., Burgay, M., et al. 2007b [arXiv:0711.04821v1] proceeding

Jansen, F., Lumb, D., Altieri, B., et al. 2001, A\&A, 365, L1

Kaplan, D. 2008, in 40 YEARS OF PULSARS: Millisecond Pulsars, Magnetars and More. AIP Conf. Proc., 983, 331

Kaplan, D. L., \& Van Kerkwijk, M. H. 2005a, ApJ, 628, L45

Kaspi, V., Isolated Neutron Stars: From the Interior to the Surface, Astorphysics \& Space Science, Springer, Vol. 308, Issue 1-4, 1-11, [arXiv:astro-ph/06103040]

Kramer, M., Stappers, B. W., Jessner, A., Lyne, A. G., \& Jordan, C. A. 2007, MNRAS, 377, 107

Kuiper, L., Hermsen, W., \& Mendez, M. 2004, ApJ, 613, 1173

Kuiper, L., Hermsen, W., den Hartog, P. R., \& Collmar, W. 2006, ApJ, 645, 556

Lyutikov, M., \& Gavriil, F. P. 2006, MNRAS, 368, 690

Mason, K. O., Breeveld, A., Much, R., et al. 2001, A\&A, 365, L36

McLaughlin, M. A., Rea, N., Gaensler, B. M., et al. 2007, ApJ, 670, 1307

Muno, M. P., Clark, J. S., Crowther, P. A., et al. 2006, ApJ, 636, L41

Muno, M. P., Gaensler, B. M., Clark, J. S., et al. 2007, MNRAS, 378, L44

Nobili, L., Turolla, R., \& Zane, S. 2008a, MNRAS, 386, 1527

Nobili, L., Turolla, R., \& Zane, S. 2008b, MNRAS, 389, 989

Pavlov, G. G., \& Bezchastnov, V. G. 2005, ApJ, 635, L61

Perna, R., \& Gotthelf, E. V. 2008, ApJ, 681, 522

Rea, N., Israel, G. L., Stella, L., et al. 2003, ApJ, 586, L65

Rea, N., Testa, V., Israel, G. L., et al. 2004, A\&A, 425, L5

Rea, N., Oosterbroek, T., \& Zane, S. 2005, MNRAS, 361, 710

Rea, N., Israel, G. L., \& Oosterbroek, T. 2007, Ap\&SS 308, 505

Rea, N., Zane, S., Turolla, R., Lyutikov, M., \& Götz, D. 2008, ApJ, 686, 1245

Schreier, E., Levinson, R., Gursky, H., et al. 1972, ApJ, 172, L79

Skinner, S. L., Perna, R., \& Zhekov, S. A. 2006, ApJ, 653, 587

Strüder, L., Briel, U., Dennerl, K., et al. 2001, A\&A, 365, L18

Testa, V., Rea, N., Mignani, R. P., et al. 2008, A\&A, 482, 607

Titarchuk, L. 1994, ApJ, 434, 570

Thompson, C., \& Duncan, R. C. 1995, MNRAS, 275, 255

Turbiner, A. V. 2007, Ap\&SS, 308, 267

Turbiner, A. V., \& Lopez-Vieyra, J. C. 2006, Phys. Rep., 424, 309

Turner, M. J. L., Abbey, A., Arnaud, M., et al. 2001, A\&A, 365, L27 van Kerkwijk, M. H., \& Kaplan, D. L. 2007, Ap\&SS, 308, 191

Woods, P. M., \& Thompson, C. 2006, Compact stellar X-ray sources, 547

Woods, P. M., Kouveliotou, C., Göğüş, E., et al. 2002, ApJ, 576, 381

Woods, P. M., Kaspi, V. M., Thompson, C., et al. 2004, ApJ, 605, 378

Woods, P. M., Kouveliotou, C., Gavriil, F. P., et al. 2005, ApJ, 629, 985

Zane, S., Turolla, R., Stella, L., \& Treves, A. 2001, ApJ, 560, 384 